ARTICLE

https://doi.org/10.1038/s41467-019-12962-4

\title{
Tsc1 represses parvalbumin expression and fast- spiking properties in somatostatin lineage cortical interneurons
}

Ruchi Malik (10) 1,2,3, Emily Ling-Lin Pai ${ }^{1,4,5}$, Anna N Rubin (10) 1,5, April M Stafford ${ }^{6}$, Kartik Angara ${ }^{6}$, Petros Minasi ${ }^{1}$, John L. Rubenstein ${ }^{1,4,5}$, Vikaas S Sohal ${ }^{1,2,3 \star} \&$ Daniel Vogt (10 ${ }^{6,7 \star}$

Medial ganglionic eminence (MGE)-derived somatostatin (SST)+ and parvalbumin (PV)+ cortical interneurons ( $\mathrm{CINs}$ ), have characteristic molecular, anatomical and physiological properties. However, mechanisms regulating their diversity remain poorly understood. Here, we show that conditional loss of the Tuberous Sclerosis Complex (TSC) gene, Tsc1, which inhibits the mammalian target of rapamycin (MTOR), causes a subset of SST+ CINs, to express PV and adopt fast-spiking (FS) properties, characteristic of PV+ CINs. Milder intermediate phenotypes also occur when only one allele of Tsc1 is deleted. Notably, treatment of adult mice with rapamycin, which inhibits MTOR, reverses the phenotypes. These data reveal novel functions of MTOR signaling in regulating PV expression and FS properties, which may contribute to TSC neuropsychiatric symptoms. Moreover, they suggest that CINs can exhibit properties intermediate between those classically associated with PV+ or SST+ CINs, which may be dynamically regulated by the MTOR signaling.

\footnotetext{
${ }^{1}$ Department of Psychiatry and UCSF Weill Institute for Neurosciences, 675 Nelson Rising Ln, San Francisco, CA 94158, USA. ${ }^{2}$ Center for Integrative Neuroscience, University of California San Francisco, 1550 4th St., San Francisco, CA 94158, USA. ${ }^{3}$ Sloan-Swartz Center for Theoretical Neurobiology, University of California San Francisco, 1550 4th St., San Francisco, CA 94158, USA. ${ }^{4}$ Neuroscience Program, UCSF, University of California San Francisco, 1550 4th St., San Francisco, CA 94158, USA. ${ }^{5}$ Nina Ireland Laboratory of Developmental Neurobiology, University of California San Francisco, 1550 4th St., San Francisco, CA 94158, USA. ${ }^{6}$ Department of Pediatrics and Human Development, 400 Monroe Ave. NW, Grand Rapids, MI 49503, USA. ${ }^{7}$ Neuroscience Program, Michigan State University, East Lansing, MI, USA. *email: Vikaas.Sohal@ucsf.edu; Vogtdan2@msu.edu
} 
T uberous sclerosis complex (TSC) is a disorder that affects multiple organ systems. Roughly half of those diagnosed with TSC have autism spectrum disorder (ASD) or intellectual disability (ID), and $\sim 90 \%$ exhibit seizures ${ }^{1-4}$. TSC is caused by mutations in the TSC1 and TSC2 genes, which encode the HAMARTIN and TUBERIN proteins, respectively ${ }^{5,6}$. HAMARTIN and TUBERIN proteins dimerize to form a protein complex that inhibits the activity of the mammalian target of rapamycin (MTOR), a protein complex that is a rheostat for energy homeostasis and is also an important regulator of protein translation $^{7}$. Multiple proteins in the TSC/MTOR signaling pathway are either high confidence ASD-causative genes or underlie disorders with high ASD coincidence ${ }^{8,9}$. This has relevance to the high rate of ASD in TSC and potentially other TSCAssociated Neuropsychiatric Disorders (TANDs), which are common in the syndrome ${ }^{10}$. Uncovering how this pathway regulates neuronal development and function is, therefore, fundamental to understanding the molecular and cellular underpinnings of ASD and complex neuropsychiatric symptoms in TSC.

Accumulating evidence suggests that neuropsychiatric disorders, such as ASD, and associated comorbidities like epilepsy, may be partially caused by changes in cortical GABAergic interneuron (CIN) function and connectivity, which leads to excitation/inhibition (E/I) imbalance in cortical circuits ${ }^{11}$. While the role of MTOR signaling and Tsc genes on excitatory neurons has been studied for some time, relatively little is known about their roles in CIN development and function ${ }^{12-14}$. CINs are the major source of cortical inhibition and are largely derived from the medial and caudal ganglionic eminences (MGE and CGE $)^{15,16}$. Parvalbumin $(\mathrm{PV})+$ and somatostatin $(\mathrm{SST})+\mathrm{CINs}$ are derived from MGE and constitute $\sim 70 \%$ of all CINs. These cells have substantially different morphological and physiological properties ${ }^{17,18}$. PV + CINs exhibit fast-spiking (FS) firing properties and synapse onto soma/axons of excitatory neurons. By contrast, SST+ CINs have regular-spiking (RS) firing properties and target the distal dendrites of excitatory neurons ${ }^{18,19}$. The difference in firing properties between SST + and PV+ CINs is dependent on the differential expression of voltage-gated ion channels. Specifically, expression of delayed rectifying potassium channels (Kv3) is critical for FS physiology of PV+ $\mathrm{CINs}^{20}$.

From their birth through maturity, CINs acquire a combination of molecular, cellular and physiological characteristics (hence referred to as cell programming). Most studies investigating MGE-derived CIN programming have largely focused on the role of transcription factors (TFs) ${ }^{21-24}$, yet little is known about how cellular signaling influences CIN development. Recent work from us and others highlighted the importance of Pten, another inhibitor of MTOR signaling that acts upstream of Tscl, on establishing proper numbers of MGE-derived CINs in the cortex ${ }^{25,26}$. Therefore, we speculate that aberrant MTOR signaling in TSC might lead to abnormalities in the development and function of MGE-derived CINs.

To investigate this, we conditionally deleted Tsc1 in MGEderived SST-lineage CINs, which allowed us to assess the impact of $T s c 1$ loss/MTOR activity starting during early post-mitotic stages. We then investigated the role of Tscl/MTOR signaling in CIN cell programming. Surprisingly, homozygous loss of Tsc1 caused SST-lineage CINs to aberrantly exhibit properties of PV+/FS CINs. In addition, this phenotype can be rescued by inhibiting MTOR during adult stages, suggesting that drugs currently being studied to treat TSC, including rapamycin derivatives, may be effective in treating TSC symptoms caused by CIN dysfunction. Overall, our findings demonstrate novel roles for $T s c 1$ in the development and function of CINs. We propose that the choice between SST + and PV+ cell programming is mediated in part by non-transcriptional processes, including cellular signaling events, suggesting a new avenue towards understanding these important cell types.

\section{Results}

Loss of Tsc1 causes ectopic PV expression in SST lineages. To test whether loss of Tsc1 in SST-expressing post-mitotic CINs alters their development, we crossed Tsc $1^{\text {floxed }}$ mice $^{27}$ and SSTIRES-Cre mice ${ }^{28}$ (SST-Cre hereafter). The Cre-dependent tdTomato reporter, $A i 14^{29}$, was included to track the Tsc1 WT, conditional heterozygous (cHet) and knockout (cKO) cells. Tsc1 transcript was absent from SST-Cre; Tsc1 cKO CINs in the neocortex at postnatal day (P) 35 (Supplementary Fig. 1a-d). At the same age, SST-Cre-lineage cHet and cKO cells in the neocortex had elevated levels of ribosomal subunit S6 phosphorylated at Serines 240 and 244 (Supplementary Fig. 1e-g), indicating increased MTOR activity. Tsc1 cKOs had normal numbers of SST-Cre-lineage CINs (tdTomato + ) in the neocortex but the cells had increased soma size (Fig. 1a-h). The laminar distribution of tdTomato + CINs was not altered by Tsc1 deletion (Supplementary Fig. 2).

SST-lineage CINs are derived from MGE progenitors that also give rise to $\mathrm{PV}+\mathrm{CINs}$. Thus, we investigated the expression of SST and PV at P35 in the neocortex. While the \% of tdTomato+ CINs that expressed SST was unchanged, there was $\sim 3$-fold increase in the \% of SST-Cre-lineage CINs that expressed PV in cKO mice (Fig. 1a-f, i, j). These data suggest that SST and PV proteins could be co-expressed in a subset of SST-Cre-lineage CINs in cKO mice. To test this, we quantified WT, Tscl cHet and cKO neocortices for SST and PV co-labeled CINs at P35 in the neocortex. While there were almost no co-labeled CINs in WTs, $\sim 2$ and $13 \%$ co-labeled CINs were observed in cHets and cKOs, respectively (Fig. 1k-q). A similar phenotype of ectopic PV expression in SST + CINs after Tscl deletion was observed in Nkx2.1-Cre; Tsc1 cKOs (Supplementary Fig. 3), which begins to express in MGE progenitors ${ }^{30}$. Together, these data suggest that Tsc1 deletion causes PV expression in a subset of SST-lineage CINs, and its deletion causes these SST + CINs to develop a dual molecular identity.

Since PV expression was elevated in a subset of SST + CINs after Tscl deletion, we wanted to assess whether MTOR activity may be different in WT SST+ and WT PV+ CINs in the neocortex. To this end, we assessed the expression of phosphorylated S6, a marker of MTOR activity, in SST + or PV+ CINs in the P35 WT neocortex. While only $20 \%$ of SST + CINs coexpressed this marker, $\sim 70 \%$ of $\mathrm{PV}+\mathrm{CINs}$ were co-labeled (Fig. 2a-g). Thus, PV + CINs normally exhibit greater MTOR activity than SST+ CINs in the neocortex.

SST CINs in Tsc1 cKOs exhibit fast-spiking properties. Next, we assessed the physiological properties of SST-lineage CINs in the Tscl cKOs. We obtained ex vivo patch clamp recordings from layer 5 SST-Cre-lineage CINs (tdTomato+) in WT, cHet and cKO mice (example cells, Fig. 3a, and current induced voltage responses, Fig. 3b). WT CINs had RS physiological properties, including strong spike frequency accommodation (SFA), wider spikes, relatively slow action potential (AP) rate of rise (max dV/ $\mathrm{dt}$ ) and relatively small fast afterhyperpolarization (fAHP) amplitudes (Fig. 3), as expected ${ }^{17,18,31}$. Consistent with the increased complexity of neuron processes promoted by increased MTOR signaling ${ }^{31,32}$, loss of Tsc1 decreased the input resistance $\left(\mathrm{R}_{\text {in }}\right)$ and produced a corresponding increase in the rheobase (current threshold) of cKO CINs (Fig. 3c, f). A reduction in $\mathrm{R}_{\text {in }}$ and an increase in rheobase predicts that Tscl loss would lower the excitability of SST+ CINs. However, Tsc1 cKO CINs had 

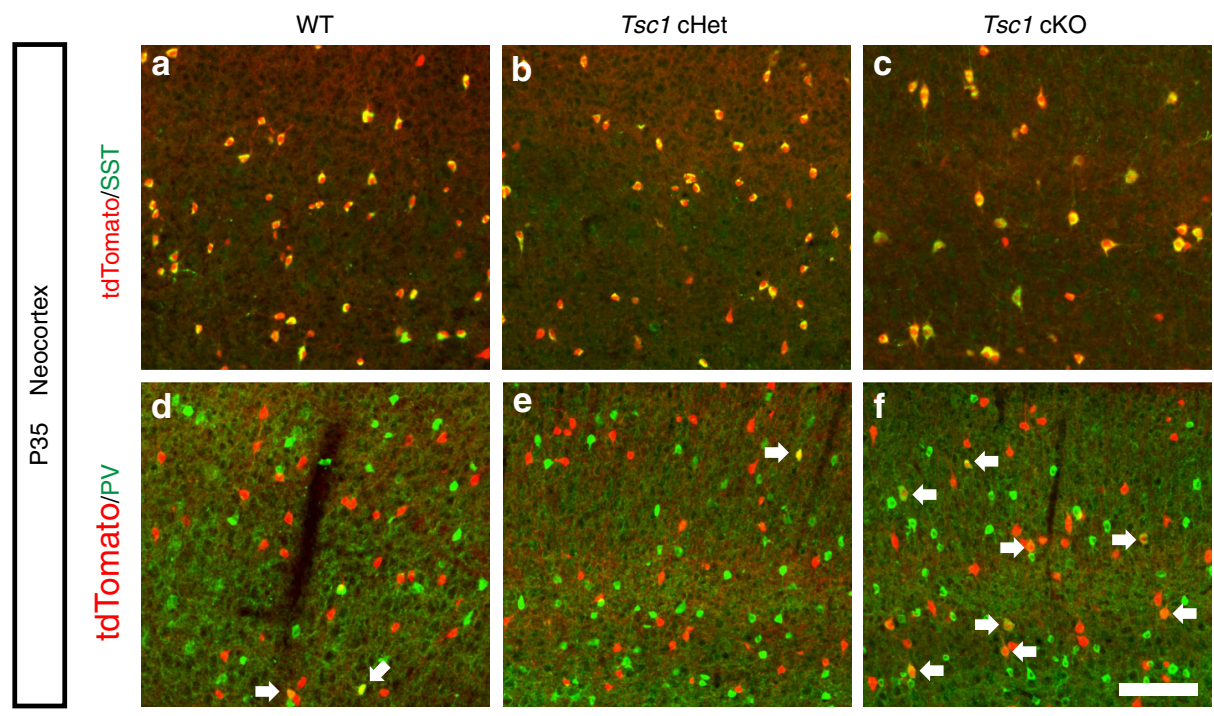

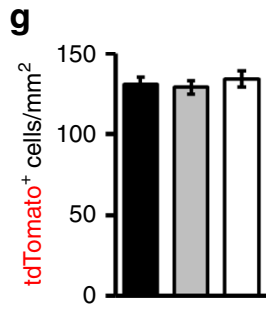

h

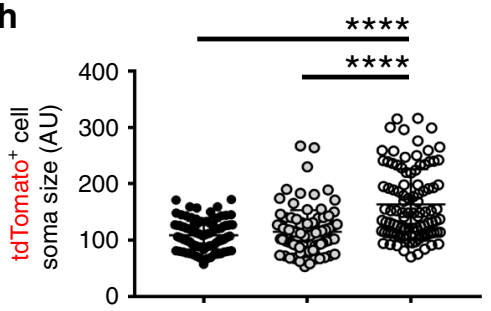

i
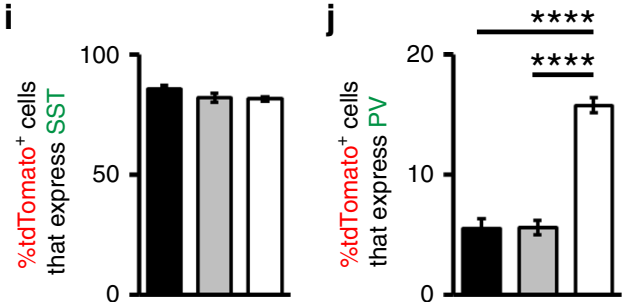

WT

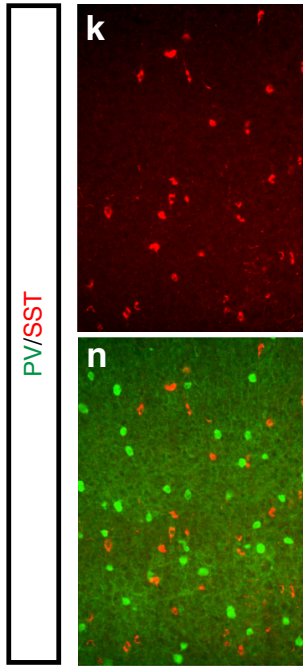

Tsc1 cHet
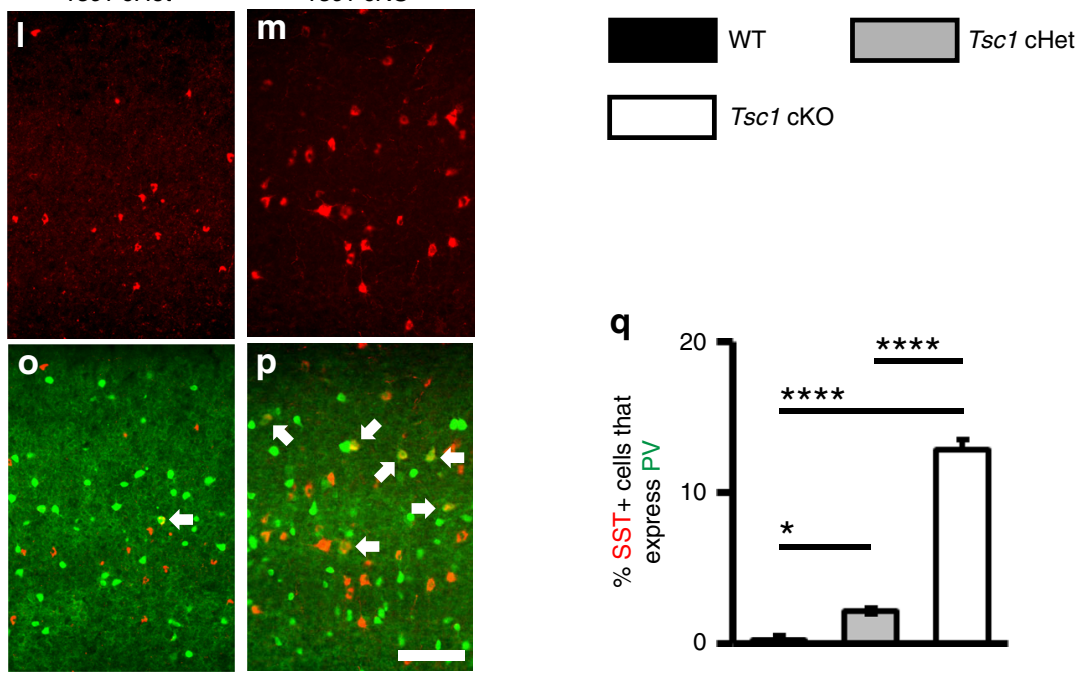

Fig. 1 Post-mitotic deletion of Tsc1 in SST-Cre-lineages results in increased PV expression. Coronal immunofluorescent images from WT, Tsc1 cHets or Tsc1 cKOs P35 neocortices, showing co-localization of tdTomato (SST-Cre-lineages) with either SST (a-c) or PV (d-f). Arrows d-f denote cells in the SST-Crelineage that express PV. $\mathbf{g}$ Quantification of the number of tdTomato+ cells $/ \mathrm{mm}^{2}$ (cell density) in the neocortex: $n=6$ (WT), 6 (cHet) and 4 (cKO) mice, One-way ANOVA $\left(F_{2,13}=0.35, P=0.7\right)$. h Quantification of tdTomato+ cell soma size: $n=4$ mice per genotype (100 cells measured from each genotype), $\left(F_{2,297}=44.9, P<0.0001\right.$; $c K O$ vs. WT and cHet, $\left.p<0.0001\right)$. (AU) arbitrary units. $\mathbf{g}$, $\mathbf{h}$ Quantification of the \%tdTomato+ cells that coexpress either SST (i) or PV (j): Chi-squared test with Yate's correction, ( $p<0.0001$, all groups); $n=6$ WT (1268 cells), $n=6 \mathrm{cHet}(1371 \mathrm{cells})$ and $n=4$ cKO (1218 cells) for SST; $n=5$ WT (1281 cells), $n=6$ cHet (1600 cells) and $n=4$ cKO (1220 cells) for PV. k-p Immunofluorescent images showing SST and PV co-labeling in the neocortex. q Quantification of the \%SST+ CINs that co-express PV: Chi-squared test with Yate's correction, (WT vs. cHet, $p=0.03$, cKO vs. WT or cHet, $p<0.0001), n=3$ mice, all groups. Data are represented as mean \pm SD in (h) and \pm SEM in all other graphs. Scale bars in $\mathbf{f}, \mathbf{p}=100 \mu \mathrm{m} .{ }^{\star} p<0.05,{ }^{\star \star \star \star} p<0.0001$. Source data are provided as a Source Data file

increased firing output (Fig. 3d, g-h). This increase was most pronounced within a subset of SST-Cre lineage CINs that shifted their physiology from RS to FS (Fig. 3h and Supplementary Fig. 4). This shift from a RS to FS phenotype was most prevalent in CINs from cKO mice (Fig. 3g, h), and was consistent with the increased \% of tdTomato + CINs expressing PV (Fig. 1). Most of the SST-lineage CINs classified as FS in Tsc1 cKOs expressed PV (Supplementary Fig. 5). While a small subset of CINs in cHet and cKO mice completely switched their physiology from RS to FS, $\sim 30 \%$; the firing and single AP properties of most SST-Cre-lineage 

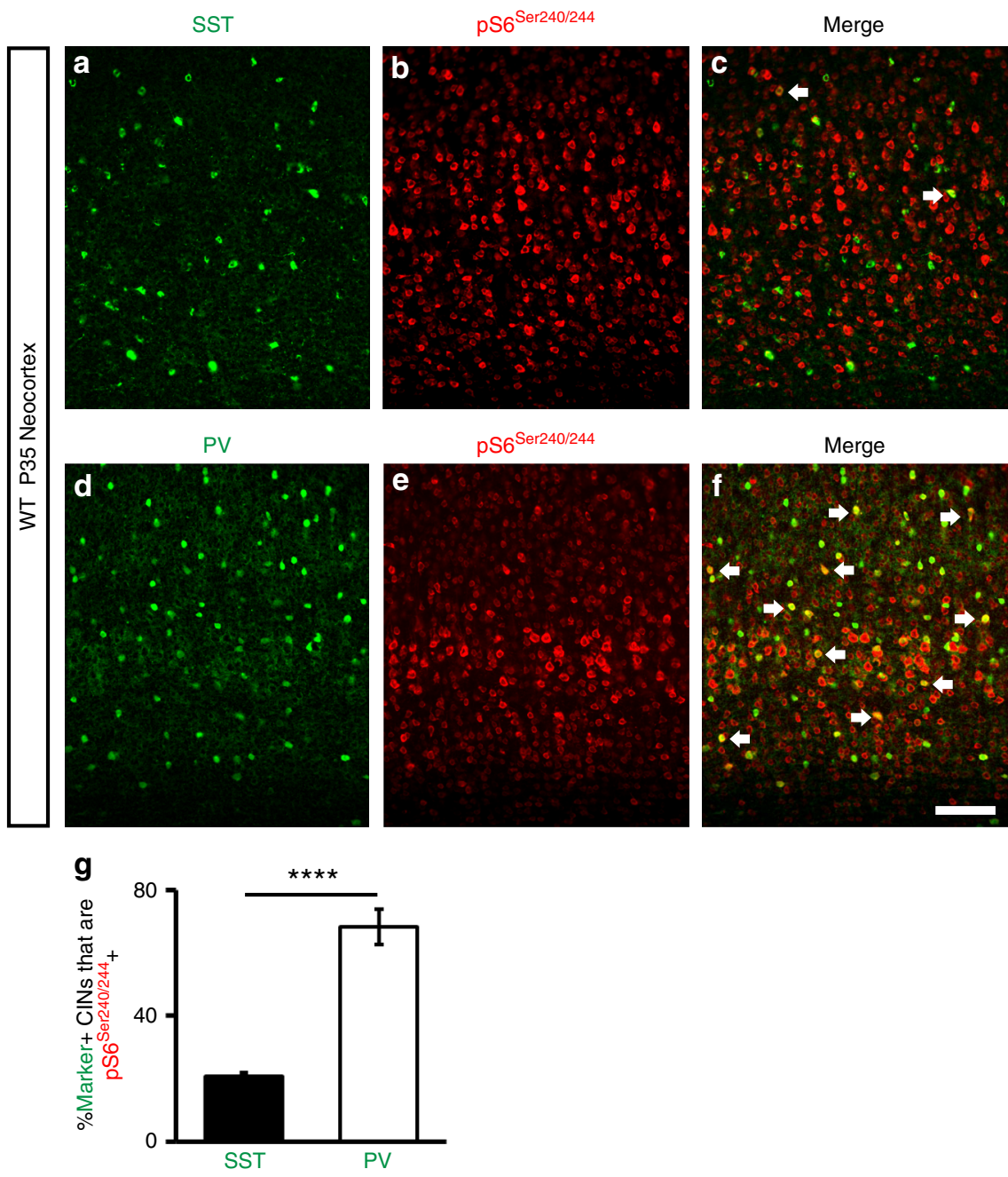

Fig. 2 The MTOR activity marker, pS6, is preferentially enriched in PV+ CINs. a-c P35 immunofluorescent images of WT neocortices co-labeled for SST and pS6. d-f Immunofluorescent co-labeling of PV and pS6 from P35 WT neocortices. Arrows denote co-labeled CINs. $\mathbf{g}$ Quantification of the \% of either $\mathrm{SST}+$ or PV $+\mathrm{CINs}$ that are pS6+: Chi-squared test with Yate's correction, ${ }^{\star \star \star *} p<0.0001$. Data are expressed as the $\pm \mathrm{SEM}, n=3$ mice/marker (145 SST cells and 208 PV cells counted). Scale bar in $\mathbf{f}=100 \mu \mathrm{m}$. Source data are provided as a Source Data file

CINs in cHet and cKO mice shifted towards a FS-like physiology. Further, the firing output and AP properties of SST + CINs with FS physiology were comparable to properties of FS PV+ CINs (Supplementary Fig. 6). Significant shifts were observed in AP threshold, AP rate of rise (max. $\mathrm{dV} / \mathrm{dt}$ ), AP duration and fAHP amplitudes (Fig. 3i-m).

Apart from differences in firing properties, SST + and PV + CINs are known to differ in their expression of Hyperpolarizationactivated cyclic nucleotide-gated $(\mathrm{HCN})$ channels $^{33}$. Compared to WT SST+ CINs, WT PV+ CINs have lower HCN channel mediated sag and rebound. However, the Tsc1 deletion mediated switch in physiology of SST + CINs was not accompanied by a change in sag and rebound (Supplementary Fig. 7). Together, these analyses showed that while loss of Tsc1 reduced the excitability of SST + CINs, their overall firing output was higher due to the increased proportion of SST + CINs with PV-like/FS properties.

Increased Kv3.1 expression in Tsc1 cKO SST CINs. Since Tsc1 deletion resulted in a shift towards FS physiology within SST-Crelineages, we asked whether Tsc1 cHets and cKOs had increased levels of the fast-inactivating Kv3.1 potassium channel. Kv3.1 expression is primarily restricted to PV $+\mathrm{CINs}$ and is the primary contributor to their fast-spiking properties (like short AP halfwidth and large fAHP) 20,34 (Supplementary Fig. 8). Indeed, we found that a similar proportion of SST-Cre-lineage CINs coexpressed Kv3.1 in cHets and cKOs (Fig. 4a, b).

To understand the effects of increased Kv3.1 channel expression on the physiological properties of SST + CINs, we used biophysical simulations in four different models of neocortical layer $5 \mathrm{SST}+\mathrm{CINs}^{35}$. Specifically, we asked if an increase in somatic Kv3.1 conductance density was sufficient to produce a transition from RS to FS properties (similar to the switch observed in Tsc1 cKOs). In all four models, increasing Kv3.1 conductance density increased the firing output ( 28\%), reduced spike frequency accommodation $(\sim 40 \%)$, reduced $\mathrm{AP}$ half-width $(\sim 32 \%)$, and increased fAHP amplitude, $(\sim 80 \%)$ (Fig. 4c-i and Supplementary Fig. 9). These results suggest that the FS properties observed in Tsc1 cKOs are due to elevated Kv3.1channels in SST-lineage CINs.

Tsc1 deletion caused an increase in pS6, PV and Kv3.1 expression in a cohort of SST-Cre-lineage CINs, and also increased their cell body (soma) size. Since SST-Cre-lineage CIN cKOs exhibited a range of soma sizes, it allowed us to assess whether cell size was correlated with the increased expression of molecular markers, i.e. pS6, PV and Kv3.1. We found a positive correlation between cell size and pS6 expression but no 
a

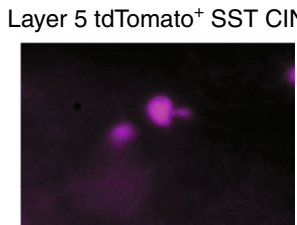

b
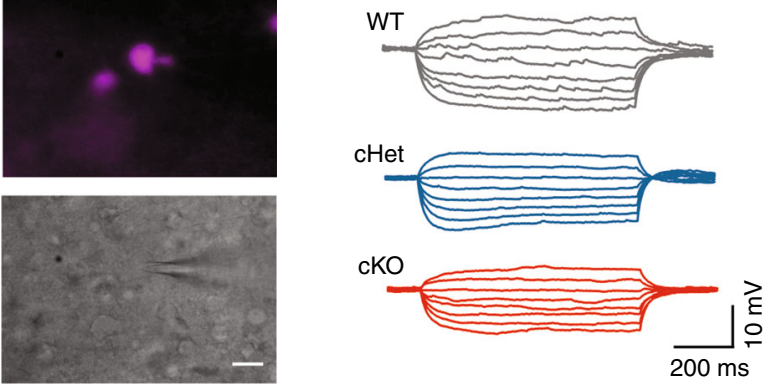

C
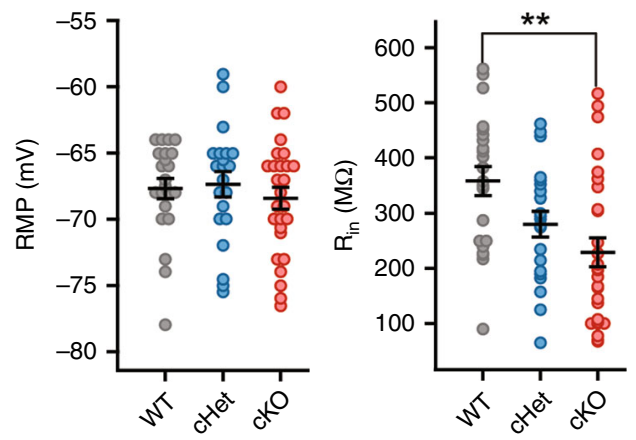

$\sqrt{3}$

WT

cHet

cKO
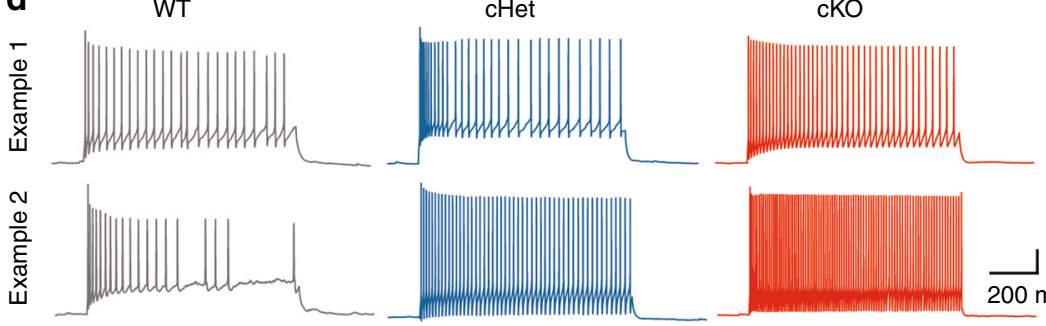

e
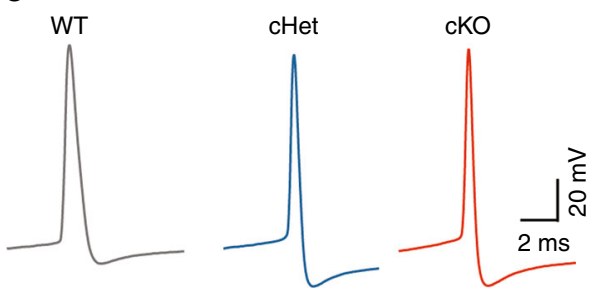

f

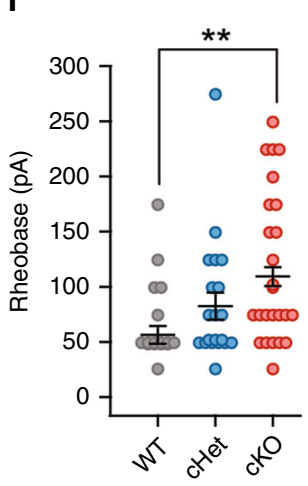

g

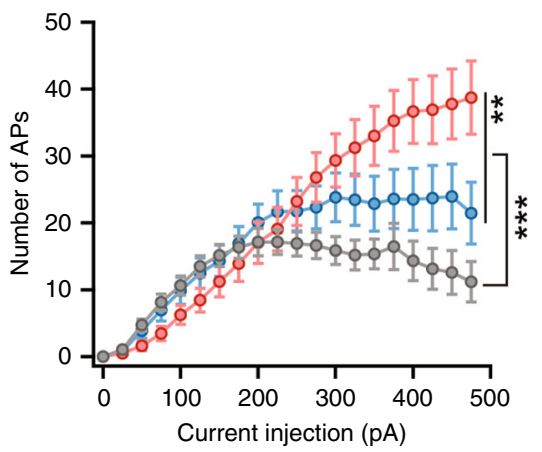

h

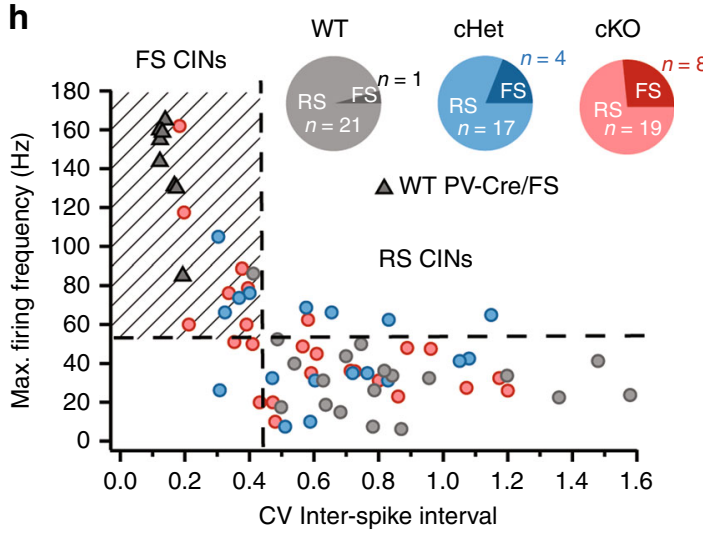

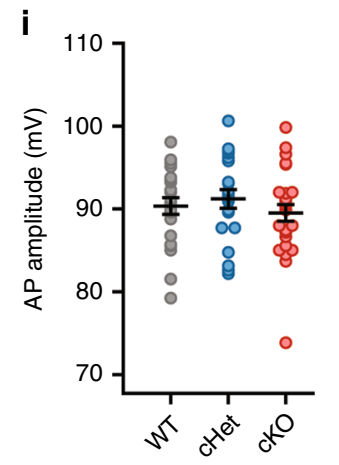

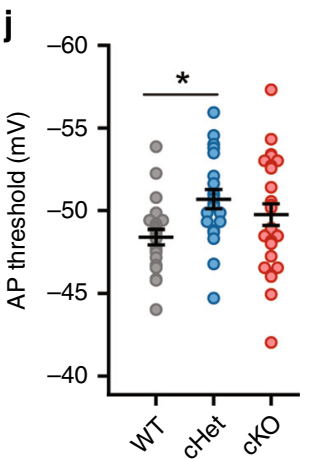

I

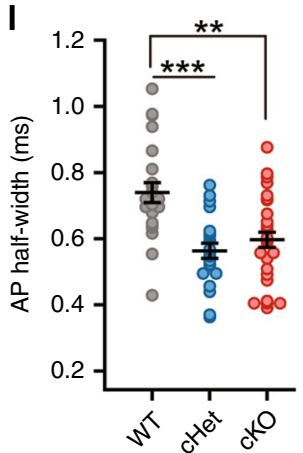

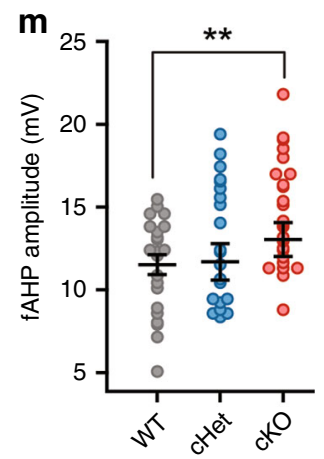

correlation between increased cell size and the expression of PV or Kv3.1, suggesting that cell size per se may not lead to the elevated PV and Kv3.1 levels (Supplementary Fig. 10).

Reduced inhibitory synaptic output in Tsc1 cKOs. Given that loss of Tsc1 affected the excitability and firing output of SST+ CINs, we wondered how these changes might affect inhibitory synaptic output onto nearby pyramidal neurons. Thus, we compared spontaneous inhibitory postsynaptic currents (sIPSCs) recorded from cortical layer 5 pyramidal neurons in WT, cHet and cKO mice (Fig. 5a). Surprisingly, the frequency and amplitude of sIPSCs was lower in cHet and cKO mice (Fig. 5c). We had expected that the increase in firing output might result in increased spontaneous inhibitory transmission. This reduction in spontaneous inhibitory output could be caused by a reduction in numbers of inhibitory synapses and/or probability of release at these synapses. Towards elucidating the respective contributions of these various possibilities, we added TTX $(1 \mu \mathrm{M})$ to the recording solution, to remove large amplitude, AP-dependent IPSCs (and therefore any effects of changes in excitability). Similar to sIPSCs, the frequency and amplitude of miniature IPSCs (mIPSCs) were lower in Tsc1 cKOs (Fig. 5b, d). There were no changes in sIPSC and mIPSC rise time and tau between 
Fig. 3 SST-Cre-lineage CINs adopt fast-spiking (PV-like) properties after Tsc1 deletion. a Fluorescence (top) and DIC (bottom) images illustrating that recordings were obtained from tdTomato $+/ \mathrm{SST}+\mathrm{CINs}$. Scale bar is $20 \mu \mathrm{m}$. $\mathbf{b}$ Example voltage traces in response to subthreshold current injections in WTs (gray), Tsc1 cHets (blue) and Tsc1 cKOs (red). c Comparison of resting membrane potential (RMP), $\left(F_{2,67}=0.41, P=0.66\right)$ and input resistance $\left(R_{\text {in }}\right),\left(F_{2,67}=6.49, P=0.002\right.$; WT vs. $\left.c K O p=0.001\right)$, One-way ANOVA with Tukey's test. d Example traces in response to suprathreshold current injections. Traces from two different cells are shown. e Example traces showing action potentials (APs). f Rheobase of SST+ CINs, One-way ANOVA with Tukey's test $\left(F_{2,67}=5.06, P=0.009 ;\right.$ WT vs. $\left.c K O, p=0.006\right)$. g Number of APs fired. Two-way ANOVA with Tukey's test, Genotype: $\left(F_{2,}\right.$ 1320 $=26.8$, $P<0.0001$ ); WT vs. cHet, and WT vs. $c K O, p<0.0001$; cHet vs. $c K O, p=0.008$ ). h Maximum firing frequency of SST+ CINs from WT, cHet and cKO mice, and PV + CINs from WT mice (gray triangles) is plotted against the coefficient of variance (CV) of inter-spike interval. Inset, proportion of SST+ CINs with regular-spiking (RS) and fast-spiking (FS) properties. $\mathbf{i}-\mathbf{m}$ Comparison of AP amplitude $(\mathbf{i}):\left(F_{2,67}=0.67, P=0.51\right), A P$ threshold $(\mathbf{j}):\left(F_{2,67}=\right.$ 3.48, $P=0.03$; WT vs. cHet $p=0.03)$, AP $\max d V / d t(\mathbf{k}):\left(F_{2,67}=6.39, P=0.002 ;\right.$ WT vs. cHet $p=0.004$, WT vs. cKO $\left.p=0.009\right)$, AP half-width (I) $\left(F_{2,67}=11.04, P<0.0001\right.$; WT vs. cHet $p=0.0001$, WT vs. $\left.c K O p=0.001\right)$; and fAHP amplitude $(\mathbf{m}):\left(F_{2,67}=5.27, P=0.007 ; W T\right.$ vs. $\left.c K O p=0.005\right)$; One-way ANOVA with Tukey's test. WT, $n=22$ cells, 4 mice; cHet, $n=21$ cells, 3 mice; cKO, $n=27$ cells, 4 mice; data are presented as mean \pm S.E.M. ${ }^{\star} p<0.05 ;{ }^{\star \star} p<0.01,{ }^{\star \star \star} p<0.001,{ }^{\star \star \star \star} p<0.0001$. Source data are provided as a Source Data file

genotypes (Supplementary Fig. 11a-d). These findings suggested that the loss of Tsc1 reduced the strength of inhibitory synaptic transmission by altering the number and/or release probability at inhibitory synapses.

While we found significant reductions in spontaneous inhibitory output to cortical pyramidal neurons, we could not estimate the specific contribution of SST + CINs to this reduction by analyzing sIPSCs and mIPSCs. To directly address this, we expressed ChR2-eYFP in SST-Cre lineage CINs (WT, cHet and $\mathrm{cKO})$. By delivering pulses of blue $(470 \mathrm{~nm})$ light, during voltageclamp recordings, we measured optogenetically-evoked IPSCs (oIPSCs) from SST-lineage CINs onto layer 5 pyramidal neurons (Fig. 5e, f). In line with the observed reduction in spontaneous inhibitory output, oIPSCs were also significantly smaller in Tsc1 cKOs (Fig. 5g); oIPSC rising slope was not altered but an increase was observed in decay tau in cHets (Supplementary Fig. 11e, f). To measure the presynaptic release probability, we measured the frequency-dependent attenuation of oIPSCs and paired-pulse ratio (PPR). The attenuation of oIPSCs in response to $20 \mathrm{~Hz}$ light pulses was significantly lower in cKOs (Fig. 5h, i). Furthermore, cKOs had significantly higher paired-pulse ratios (PPRs) (Fig. 5j). These results suggest that Tsc1 deletion decreases the evoked inhibitory output at least in part by reducing presynaptic release probability in SST-lineage CINs.

The axons of PV+/FS CINs target the soma and axon initial segment of pyramidal neurons, while the axons of SST + CINs target their distal dendrites ${ }^{18,19}$. Thus, we tested whether Tsc1 deletion caused aberrant axon targeting (proximal dendrite/soma vs. distal dendrite) in a subset of SST-lineage CINs. For this, we obtained paired patch clamp recordings from connected layer 5 pyramidal neurons (Pyr) and tdTomato+ SST-Cre-lineage CINs (Fig. 6a) and analyzed the unitary IPSCs (uIPSCs) in postsynaptic Pyr neurons evoked by action potentials in presynaptic SST+ CINs (Fig. 6b). Since uIPSCs generated in response to SST+CINs arrive at the distal dendrites, the amplitude and kinetics of these currents are significantly different from IPSCs originating near the soma of pyramidal neurons ${ }^{36}$. To validate this, we compared the properties of $\mathrm{CIN} \rightarrow$ Pyr uIPSCs in $\mathrm{PV}$-Cre-lineage ${ }^{37} \mathrm{CIN}$ pyramidal neuron vs. SST-Cre-lineage CIN-pyramidal neuron pairs. In our recordings from WT mice, all $P V$-Cre lineage CINs had FS physiology and all SST-Cre lineage CINs had RS physiology (Fig. 6c). A comparison of the kinetics of SST $\rightarrow$ Pyr uIPSCs and PV $\rightarrow$ Pyr uIPSCs in WT mice showed that the uIPSC amplitude, rising slope, and total charge were all significantly larger for $\mathrm{PV} \rightarrow \mathrm{Pyr}$ pairs than $\mathrm{SST} \rightarrow \mathrm{Pyr}$ pairs (Fig. 6d-f). Next, we recorded SST $\rightarrow$ Pyr uIPSCs in cKO mice. As expected, most SST-Cre-lineage CINs in cKOs had RS properties but a subset had FS properties (Fig. 6c). Strikingly, in our paired recordings from cKOs, the amplitude, kinetics and total charge uIPSCs did not differ for RS/SST $\rightarrow$ Pyr vs. FS/
SST $\rightarrow$ Pyr pairs (Fig. $6 \mathrm{~d}-\mathrm{f}$ ). These results suggest that while Tsc1 deletion caused a switch in physiological properties and molecular identity of SST-Cre lineage CINs towards a PV-like/ FS physiology, it did not affect their axonal targeting. Notably, morphological reconstructions of SST + CIN axons showed that loss of Tsc1 was not accompanied by changes in axonal targeting and branching (Supplementary Fig. 4). These findings, together with the lower presynaptic release probability, may explain why, despite the increased firing output and shift towards PV-like phenotypes, inhibitory output mediated by SST + CINs is reduced in Tsc1 cKOs.

Cell autonomous role for Tsc1 in PV CINs. Since Tsc1 cKOs exhibited reduced inhibitory tone in the neocortex (Fig. 5), we asked if the increased PV expression in SST-Cre-lineages is cell autonomous, as PV expression can be modulated by circuit activity $^{38,39}$. We utilized an MGE transplantation assay that introduces MGE progenitors in small numbers to a WT cortex for in vivo maturation ${ }^{40}$. MGE tissue from WT, Tsc1 Floxed/+ or Tsc1 ${ }^{\text {Floxed/Floxed }}$ was isolated from E13.5 embryos; dissociated cells were transduced with DlxI12b-Cre expressing lentiviruses (Fig. 7a). The DlxI12b enhancer biases expression to GABAergic neurons and has been used to express genes efficiently in developing and mature $\mathrm{CINs}^{25,41,42}$. These MGE cells were then transplanted into WT neonatal cortex and allowed to develop. After 35 days post-transplant (DPT), we assessed the proportion of transplanted cells (tdTomato+) that expressed PV. Tsc1 cKO cells had increased soma size and $\sim 2$-fold increase in PV expression compared to WT (Fig. 7b-d, f, g). Finally, we expressed the human TSC1 gene from the same lentiviral vector, to test if human TSC1 could compliment the mouse Tsc1 depleted CINs and found that it was able to rescue the increase in soma size and PV expression (Fig. 7e-g).

Rapamycin decreases PV and FS properties in Tsc1 cKOs. We hypothesized that Tsc1 normally represses PV-like/FS properties in SST+ CINs by inhibiting MTOR activity. We thus administered the MTOR inhibitor, rapamycin, to test whether this treatment might reverse/rescue the effects of Tsc1 loss in young adult (eight-week old) mice. Tsc1 cHets, and cKOs were treated with rapamycin or vehicle starting at eight weeks of age, for five consecutive days (Fig. 8a). A day after the last dose, mice were assessed for soma size, PV and Kv3.1 expression, and electrophysiology. To assess rapamycin's efficacy, we probed for pS6 in tdTomato+ CINs. Vehicle-treated Tsc1 cKO CINs had increased co-expression of pS6 compared to the cHet groups and rapamycin treatment significantly reduced these levels (Supplementary Fig. 12a-e). Notably, rapamycin treatment did not alter the 


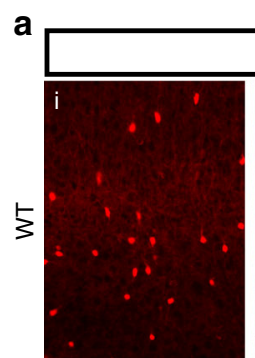

Kv3.1/tdTomato
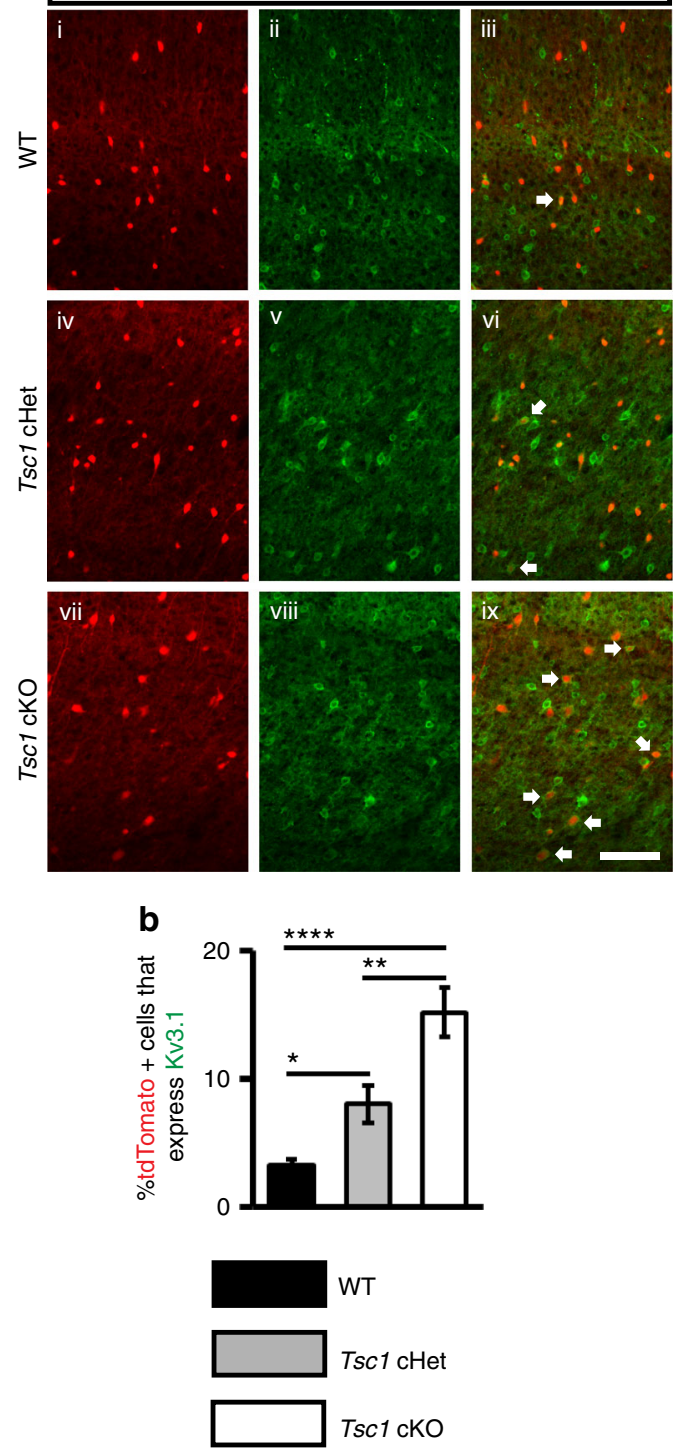

C
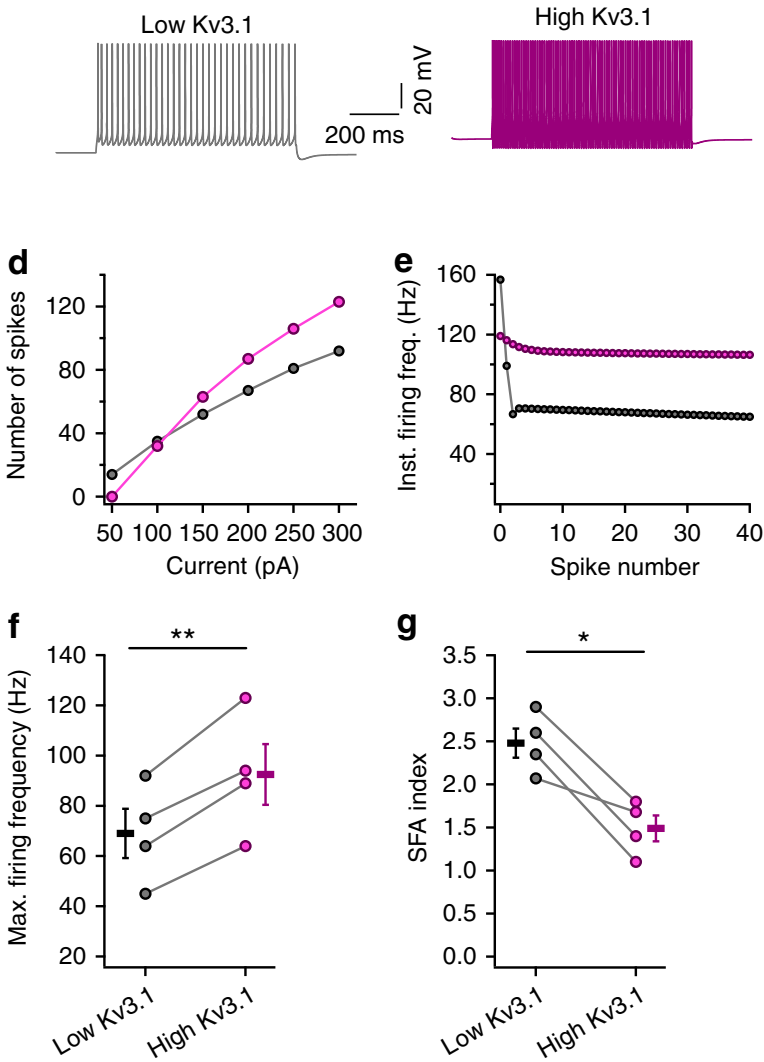

g

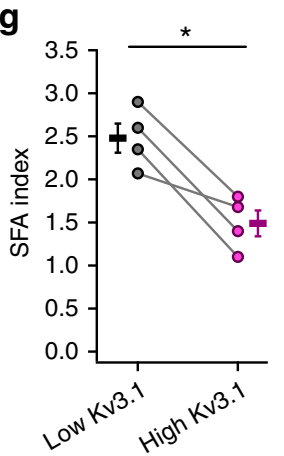

h

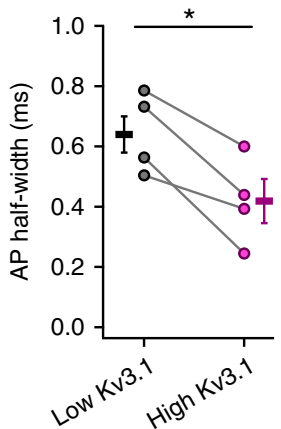

i

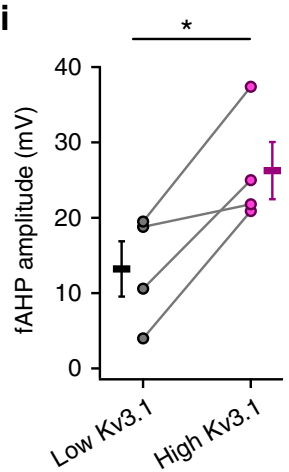

Fig. 4 Increased Kv3.1 channel expression in SST-Cre-lineage CINs underlie FS properties. a P35 coronal sections showing immunofluorescent labeling of Kv3.1 and tdTomato+ cells from SST-Cre-lineages in the neocortices of WT (i-iii), Tsc1 cHets (iv-vi) and cKOs (vii-ix). Arrows denote co-labeled cells. Scale bar is $100 \mu \mathrm{m}$. b Quantification of the \%tdTomato+ cells expressing Kv3.1: Chi-squared test with Yate's correction, WT vs. cHet, $p=0.02$ or cKO, $p<0.0001$, and cHet vs. cKO, $p=0.008, n=3$, all groups ( $405 \mathrm{WT}, 441 \mathrm{cHet}$ and $446 \mathrm{cKO}$ cells counted). c Example firing traces from a model of a layer 5 SST+ CIN with low expression of Kv3.1 (grey) and high expression of Kv3.1 (purple). d, e Firing data from one representative model is shown. Increasing the conductance of Kv3.1 channel increases the number of APs fired in response to depolarizing current injections (d) and decreases the change in instantaneous firing frequency during a train of APs (e). Increasing the Kv3.1 conductance in four different models of SST+ CINs increases the maximum firing frequency: paired two-tailed $t$-test $\left(t_{3}=8.1, p=0.003\right)(\mathbf{f})$ and decreases the spike frequency accommodation: SFA; paired two-tailed $t$-test $\left(t_{3}=4.9\right.$, $p=0.016)(\mathbf{g})$. Increasing Kv3.1 conductance in models of SST+ CINs decreases the duration of APs: paired two-tailed $t$-test $\left(\mathrm{t}_{3}=4.7, p=0.018\right)(\mathbf{h})$ and increases the fAHP: paired two-tailed $t$-test $\left(t_{3}=3.8, p=0.03\right.$ ) (i). Data are presented as mean \pm S.E.M. ${ }^{\star} p<0.05,{ }^{\star \star} p<0.01,{ }^{\star \star \star \star} p<0.0001$. Source data are provided as a Source Data file

cell density of tdTomato+ CINs or those expressing SST (Supplementary Fig. 12f, g).

Vehicle treated cKO CINs exhibited increased soma size as well as increased $\mathrm{Kv} 3.1$ and PV expression compared to the cHet groups (Fig. 8b, c, f, g, j-l). Rapamycin treatment significantly reduced the soma size of SST-lineage CINs in cKOs and decreased Kv3.1 and PV expression, however, did not alter soma size or Kv3.1 and PV expression in SST-Cre-lineage cHets (Fig. 8d, e, h-l). In addition, we assessed whether these cellular and molecular phenotypes changed after analyzing rapamycintreated mice 5 days after discontinuing the drug. While there was no gross change in soma size 5 days after discontinuing rapamycin there were increased numbers of $\mathrm{Kv} 3.1+$ and $\mathrm{PV}+$ CINs (Supplementary Fig. 13a-c), suggesting that Kv3.1 and PV expression is dynamic with changes in MTOR activity. Using a chemogenetic approach, we tested whether PV expression in SST + CINs is linked to neuronal excitability. Adult WT SST-Cre mice expressing either Gq-DREADD-mCherry or mcherry were 
a

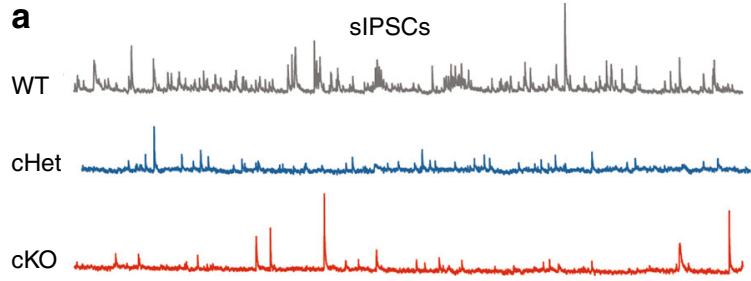

C

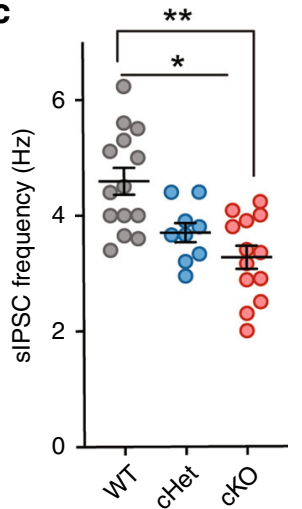

e

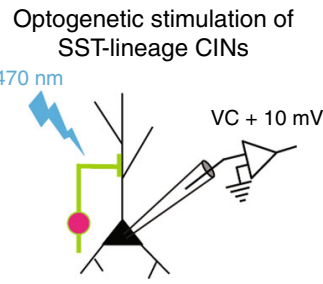

h

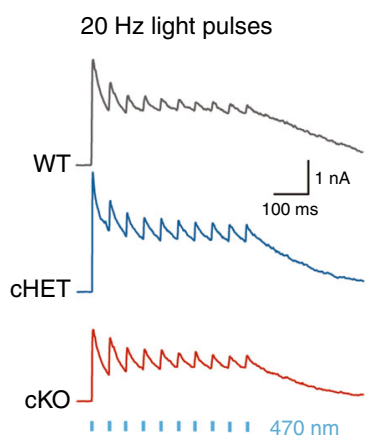

$\mathbf{f}$

i

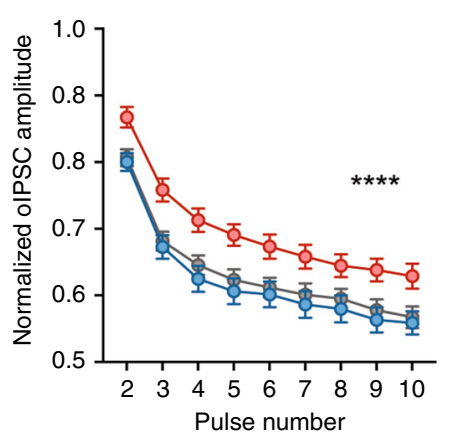

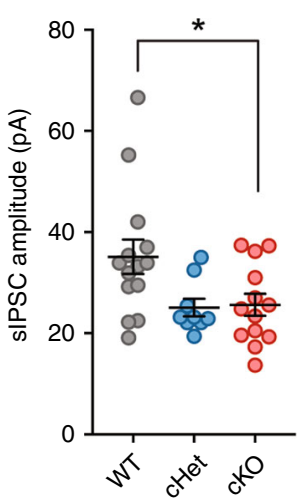

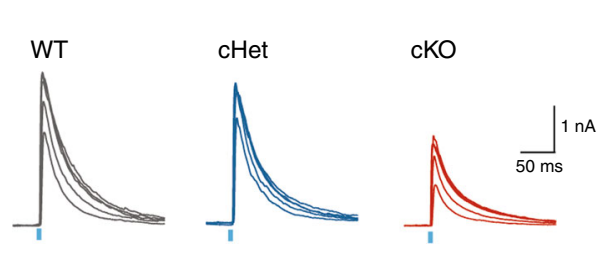

mIPSCs
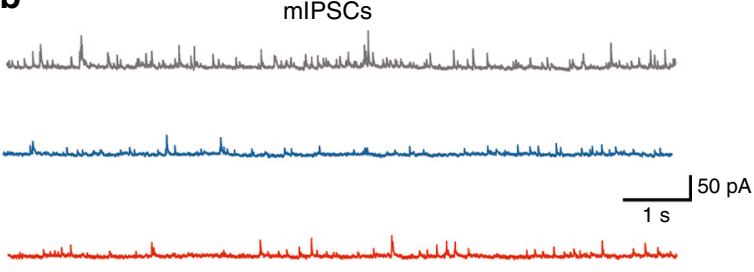

d

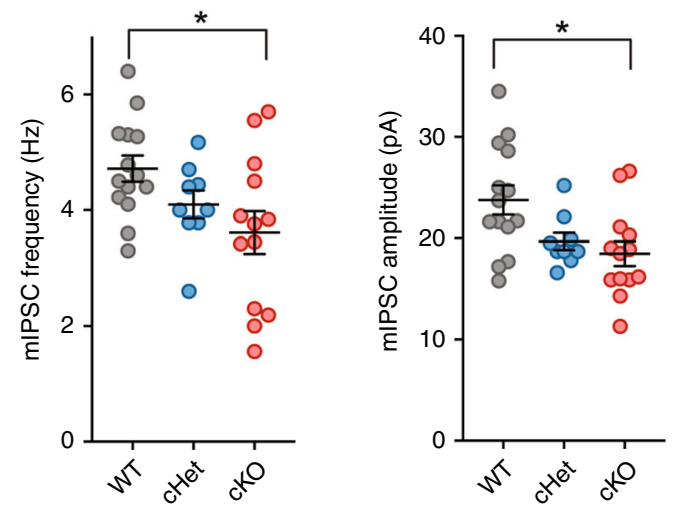

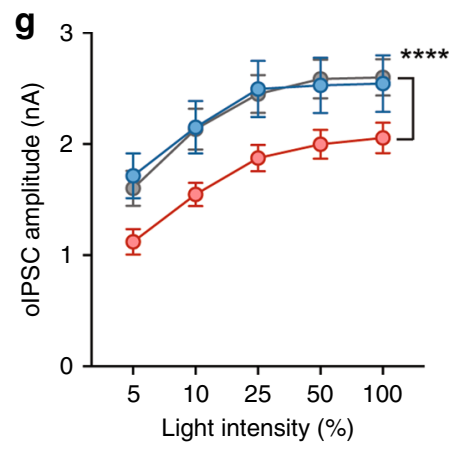

j

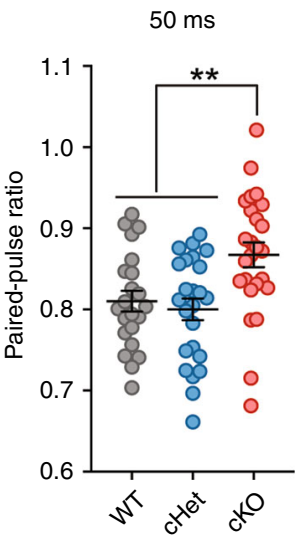

Fig. 5 Reduced inhibitory output of SST+ CINs in Tsc1 cKOs. Example traces showing sIPSCs (a) and mIPSCs (b) recorded from pyramidal neurons in WTs (grey), cHets (blue) and cKOs (red). Comparison of the frequency and amplitude of sIPSCs (c) and mIPSCs (d) (WT, $n=14$ cells, 3 mice; cHet, $n=9$ cells, 2 mice; $c K O, n=13$ cells, 3 mice). One-way ANOVA with Tukey's test, sIPSC Frequency: $\left(F_{2}, 33=11.16, P=0.0002 ;\right.$ WT vs. cHet $p=0.02 ;$ WT vs. cKO $p=0.0002) ;$ sIPSC Amplitude: $\left(F_{2}, 33=4.3, P=0.02 ;\right.$ WT vs. $\left.c K O p=0.03\right)$. mIPSC Frequency: $\left(F_{2}, 33=3.2, P=0.04 ;\right.$ WT vs. cKO $\left.p=0.04\right) ; \mathrm{mIPSC}$ Amplitude: $\left(F_{2,33}=5.1, P=0.01\right.$; WT vs. $\left.c K O p=0.01\right)$. e Voltage clamp recordings were obtained from pyramidal neurons while activating ChR2expressing SST+ CINs. f Example optogenetically-activated IPSCs (oIPSCs) recorded in WTs (21 cells, 3 mice), cHets (24 cells, 3 mice) and cKOs ( 25 cells, 3 mice) in response to blue light $(470 \mathrm{~nm}$ ) flashes of increasing light-intensity. $\mathbf{g}$ Average oIPSC amplitudes in response to light pulses of increasing intensity. Two-way ANOVA with Tukey's test $\left(F_{2,335}=16.19, P<0.0001\right.$; WT and cHet vs. cKO $\left.p<0.0001\right)$. $\mathbf{h}$ Example olPSCs in response to $20 \mathrm{~Hz}$ light pulse trains. i Attenuation in oIPSC amplitude during repeated activation of SST+ CINs by $20 \mathrm{~Hz}$ light pulse trains. Two-way ANOVA with Tukey's test $\left(F_{2,612}=56.6, P<0.0001 ; W T\right.$ and cHet vs. $\left.c K O p<0.0001\right)$. j Paired-pulse ratio of olPSCs in response to pairs of light pulses. One-way ANOVA with Tukey's test, $100 \mathrm{~ms}$ inter-pulse interval: $\left(F_{2,67}=12, p=0.0002\right.$; WT vs. cKO $p=0.002$; cHet vs. cKO $\left.p<0.0001\right)$ and 50 ms inter-pulse interval: $\left(F_{2,67}=7.4, P=0.001\right.$; WT vs. $c K O p=0.002$; cHet vs. $\left.c K O p=0.008\right)$. Data are presented as mean \pm S.E.M. ${ }^{\star} p<0.05,{ }^{\star \star} p<0.01,{ }^{\star \star \star \star} p<0.0001$. Source data are provided as a Source Data file 
a
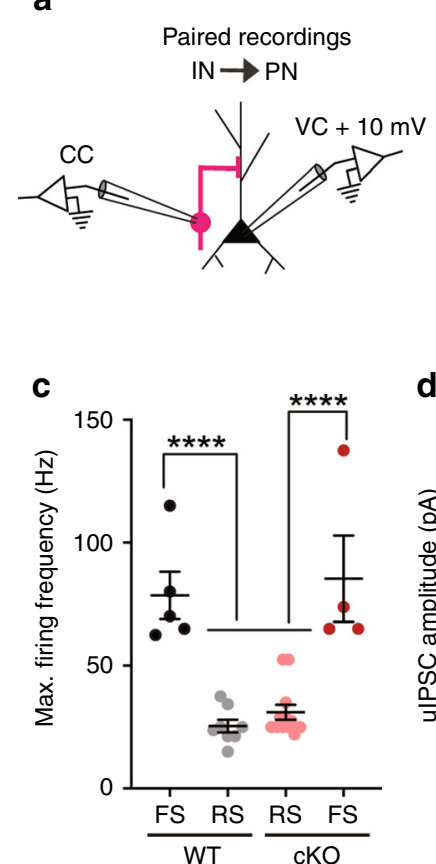

Layer 5 tdTomat+ CIN and tdtomat-Pyr neuron

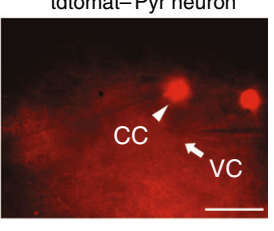

d

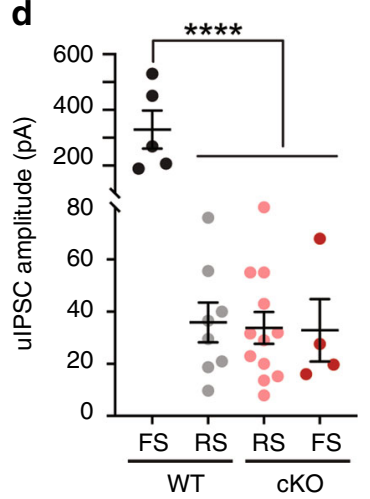

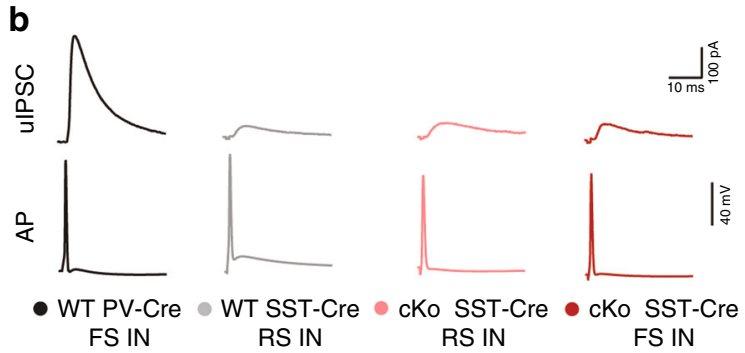

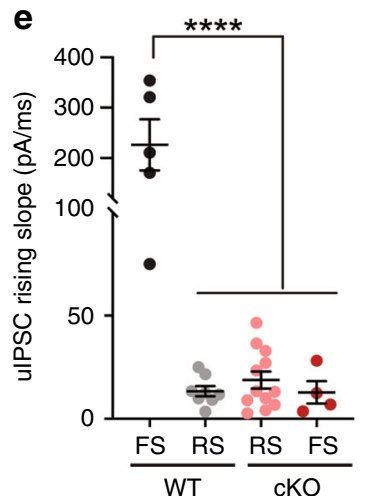

f

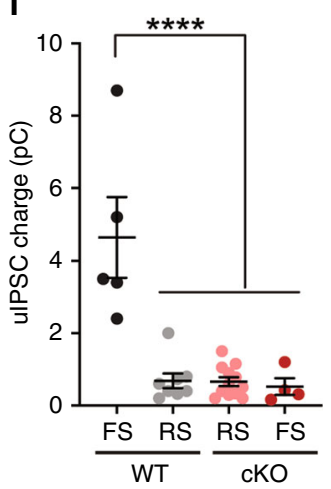

Fig. 6 FS/SST-Cre CINs in Tsc1 cKOs send their outputs to distal dendrites of pyramidal neurons. a Left, Experimental design: Simultaneous recordings were obtained from connected layer 5 tdTomato $+\mathrm{CINs}$, (current clamp, CC) and pyramidal neurons, (voltage clamp, VC), +10 mV. Right, representative image showing patch pipettes targeting tdTomato $+\mathrm{CIN}$ (arrowhead) and tdTomato- pyramidal neuron (arrow). Scale bar is $50 \mu \mathrm{m}$. b Example unitary inhibitory post synaptic currents (uIPSCs) recorded in pyramidal neurons generated in response to action potentials (AP) in WT FS/PV-Cre CINs (black, 5 pairs), WT RS/SST-Cre CINs (grey, 8 pairs), cKO RS/SST-Cre CINs (salmon, 12 pairs) and cKO FS/SST-Cre CINs (red, 4 pairs). c Maximum firing frequency of four groups of CINs is compared: One-way ANOVA with Tukey's test: $\left(F_{3,25}=20, P<0.0001 ; \mathrm{FS}\right.$ vs. RS $\left.p<0.0001\right)$. d-f uIPSC amplitude, rising slope and total charge is compared. One-way ANOVA with Tukey's test, uIPSC amplitude (d): $\left(F_{3,25}=29, P<0.0001\right.$; WT FS/PV-Cre vs. all groups $\left.p<0.0001\right)$; rising slope $(\mathbf{e}):\left(F_{3,25}=28.11, P<0.0001 ;\right.$ WT FS/PV-Cre vs. all groups $\left.p<0.0001\right) ;$ and total charge $(\mathbf{f}):\left(F_{3,25}=18, P<0.0001 ;\right.$ WT FS/PV-Cre vs. all groups $p<0.0001)$. Data are presented as mean \pm S.E.M. ${ }^{\star \star \star \star} p<0.0001$. Source data are provided as a Source Data file

given daily injections of clozapine- $\mathrm{N}$-oxide ( $\mathrm{CNO}, 3 \mathrm{mg} / \mathrm{Kg}$ dose) for 5 days. Interestingly, Gq-DREADD expressing SST+ CINs had significantly lower expression of PV in comparison to the control group (Supplementary Fig. 14). In general, these results suggest that neuronal excitability and cellular signaling events can dynamically modulate the molecular programming of CINs.

Next, we tested whether the rapamycin-mediated reduction in PV expression was accompanied by a reduction in FS physiological properties within cKO SST-Cre-lineage CINs. Rapamycin treatment did not change the $\mathrm{R}_{\mathrm{in}}$, firing output or AP properties of cHet CINs (Fig. 9e, g, h, Supplementary Fig. 14). However, in the cKO group, rapamycin treatment increased $\mathrm{R}_{\mathrm{in}}$, reduced the rheobase and increased spike frequency accommodation (SFA) (Supplementary Fig. 15). Furthermore, rapamycin reduced the firing output and decreased the fraction of SST-Crelineage CINs that were classified as FS in cKOs (Fig. 9f, i, j). Notably, even in rapamycin-treated cKO mice, the fraction of FS SST-Cre-lineage CINs was higher than the fraction of FS SST-Crelineage FS CINs in WT mice (Fig. 3h, inset). Rapamycintreatment failed to reverse some effects of Tsc1 loss, including altered AP properties in cKOs (Supplementary Fig. 15). Overall, the 5-day rapamycin treatment in adult $\mathrm{cKO}$ mice decreased the aberrant PV expression and partially reversed shifts towards FS phenotypes in SST-Cre-lineage CINs.

\section{Discussion}

Imbalances in neuronal excitation/inhibition (E/I) are implicated in neuropsychiatric disorders ${ }^{11}$, and changes in neuronal numbers, synaptic connectivity and intrinsic properties of GABAergic interneurons are, sometimes as a result of compensatory mechanisms, critical contributors to shifts in E/I balance ${ }^{25,43-46}$. TSC-associated neuropsychiatric disorders (TANDs) are common manifestations of the disorder ${ }^{10}$ but the mechanisms underlying TANDs are poorly understood. In this study, we found that deletion of the TSC causative gene, Tsc1, induced molecular and physiological changes in CINs that may underlie some neuropsychiatric symptoms and seizures observed in TSC (model, supplementary Fig. 16). First, loss of Tsc1 in SST-lineage CINs altered the programming of PV and Kv3.1 expression and induced FS properties. This reveals a novel mechanism involved in regulating the programming of CINs. Second, loss of Tsc1 in SST-lineages resulted in a neocortex with less synaptic inhibition onto deep layer pyramidal neurons, which may contribute to the higher seizure susceptibility observed in TSC. Third, altered programming could be partially reversed by rapamycin administration in adulthood, suggesting that these phenotypes remain plastic in post-mitotic CINs and could provide insights into potential therapeutic mechanisms to treat TSC.

Previous studies in a mouse model of TSC emphasized that dysregulation of MTOR signaling primarily affects excitatory circuits $^{12}$, and alters E/I balance through effects on excitatory synapses. While one report examined CINs with regards to TSC $^{47}$, little was known about changes in inhibitory circuits. In this context, we assessed cellular and physiological properties of CINs and inhibitory synapses in Tscl cHets and cKOs. We discovered that loss of Tsc1 in SST-lineages resulted in aberrant expression of PV and the voltage-gated potassium channel, Kv3.1, in a subset of SST-lineage CINs. This, in turn, shifts their physiological properties from RS to a continuum encompassing both RS and FS properties. Notably, not all SST-lineage CINs adopted these properties, showing that some are less susceptible. This is 
a

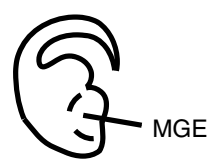

Tsc1 $^{+/+} ;$Ai14 ${ }^{\text {Floxed/+ }}$

or

Tsc1 ${ }^{\text {Floxed/++ }}$; Ai14 Floxed/+

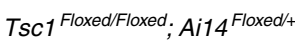

Transduce MGE cells with

DIxl12b-Cre lentiviruses

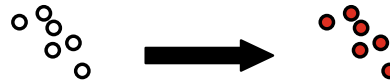

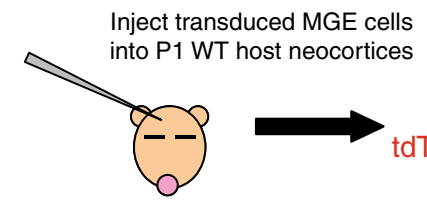

Assess cell fate of dTomato +cells at 35 DPT

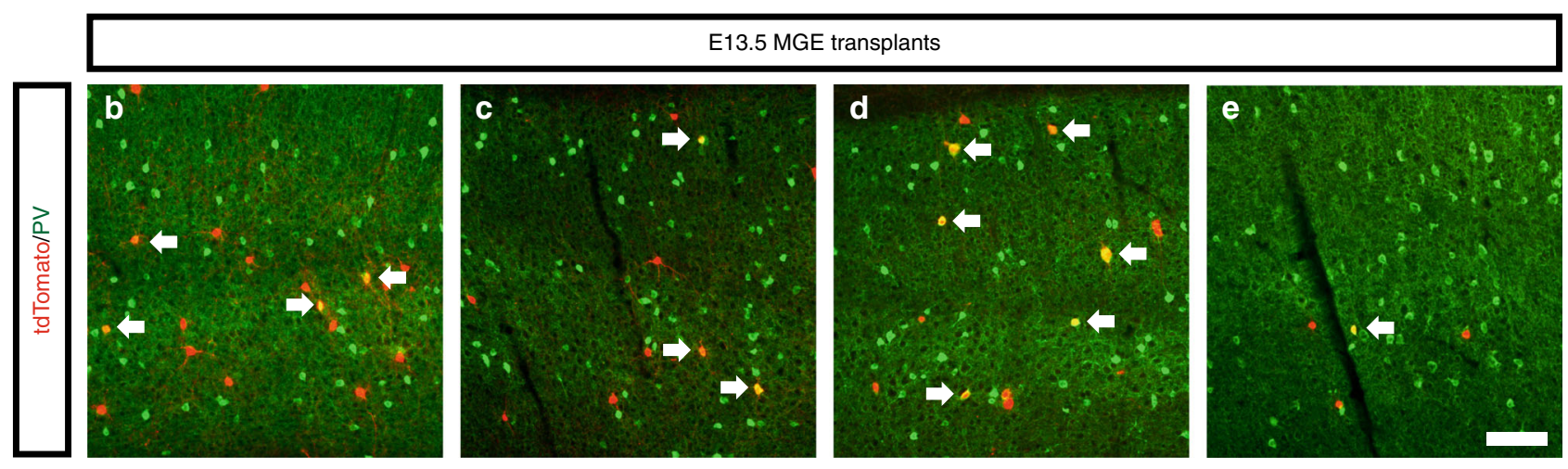

f

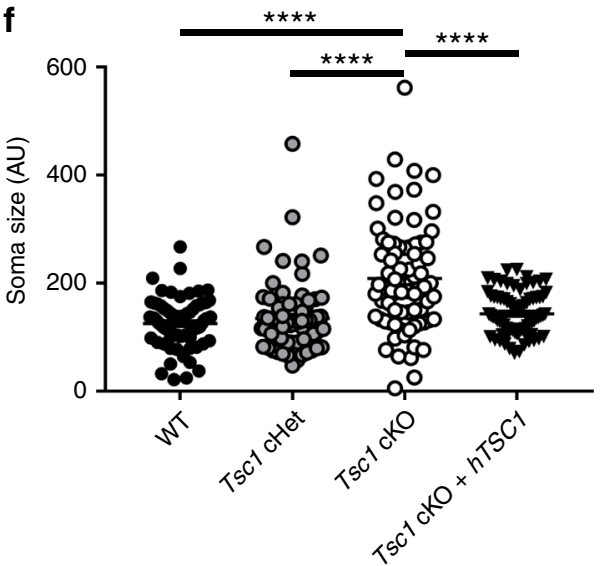

g

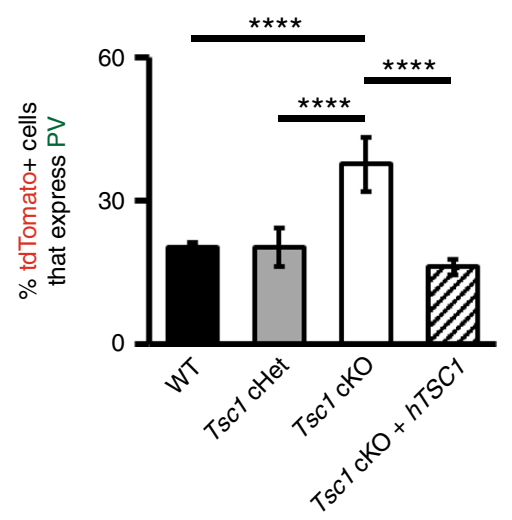

Fig. 7 Cell autonomous increase in PV expression in Tsc1 null MGE-derived CINs. a Schema depicting the transplantation procedure. E13.5 Ai14Floxed/+ MGE

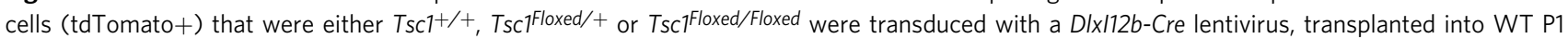
neocortices, then developed for 35 days post-transplant (DPT). Coronal immunofluorescent images of neocortices transplanted with Tsc1+/+ (WT), TsciFloxed/+ (cHet) or Tsc7Floxed/Floxed (cKO) MGE cells show co-expression of tdTomato and PV (b-d). In addition, human TSC1 was expressed in Tsc7Floxed/ Floxed (cKO) MGE cells and assessed in the same manner (e). Arrows point to co-expressing cells. Quantification of soma size of the tdTomato+ cells (f): One-way ANOVA, $\left(F_{3,296}=24.1, P<0.0001\right.$; $c K O$ vs. all groups, $\left.p<0.0001\right), n=3$ mice (25 cells counted from each). (AU) arbitrary units. Quantification of the proportion of tdTomato+ cells that express PV (g): Chi-squared test with Yate's correction, (cKO vs. all groups, $p<0.0001$ ), $n=3$, all groups (330 WT, $821 \mathrm{cHet}, 913 \mathrm{cKO}$ and $317 \mathrm{cKO} / \mathrm{hTSC1}$ cells counted). Data are represented as mean \pm SD in (f) and \pm SEM in $(\mathbf{g})$. Scale bar in (e) $=100 \mu \mathrm{m}$. ${ }^{\star \star \star \star} p<$ 0.0001. Source data are provided as a Source Data file

particularly interesting as recent data has shed new light on subpopulations of SST + CINs, with distinct molecular identities and functions ${ }^{48-50}$.

In contrast to our findings, another group deleted Tsc1 using the same SST-Cre line and a PV-Cre line but found no physiological phenotypes ${ }^{51}$, concluding that most TSC phenotypes arise from excitatory pyramidal neurons. One possibility is that these disparities could have arisen from each of our groups using different mouse background strains, C57BL/6 vs. CD-1, which could suggest that some genetic modifiers, i.e. protective and/or exacerbating, may exist that could be therapeutic targets for TSC. Moreover, studies into whether distinct cohorts of SST-lineage CINs are sensitive to MTOR signaling may help elucidate novel molecular and/or functional properties. Notably, we discovered that $\sim 20 \%$ of WT SST + CINs have observable pS6 expression (Supplementary Fig. 1) which may be a distinct susceptible cohort when Tsc1 is lost. While the phenotypes were milder, Tsc1 cHets exhibited some of the same changes observed in Tscl cKOs. Finally, TSC1/2 homozygous mutants have been identified in neural progenitors via a combination of germline and somatic mutations in a TSC patient ${ }^{52}$, potentially suggesting that some phenotypes we observed in Tsc1 $\mathrm{cKO}$ mice may also be relevant to some cases of TSC.

This study suggests new ideas about how some aspects of CIN cell programming and function are regulated, particularly in postmitotic/maturing CINs. Previous studies identified TFs that are 
a

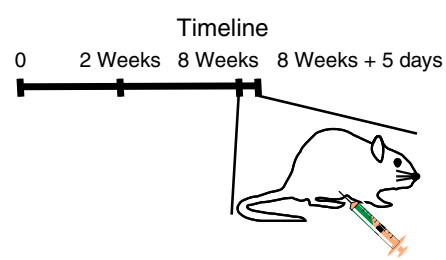

nject vehicle or Rapamycin daily for 5 days
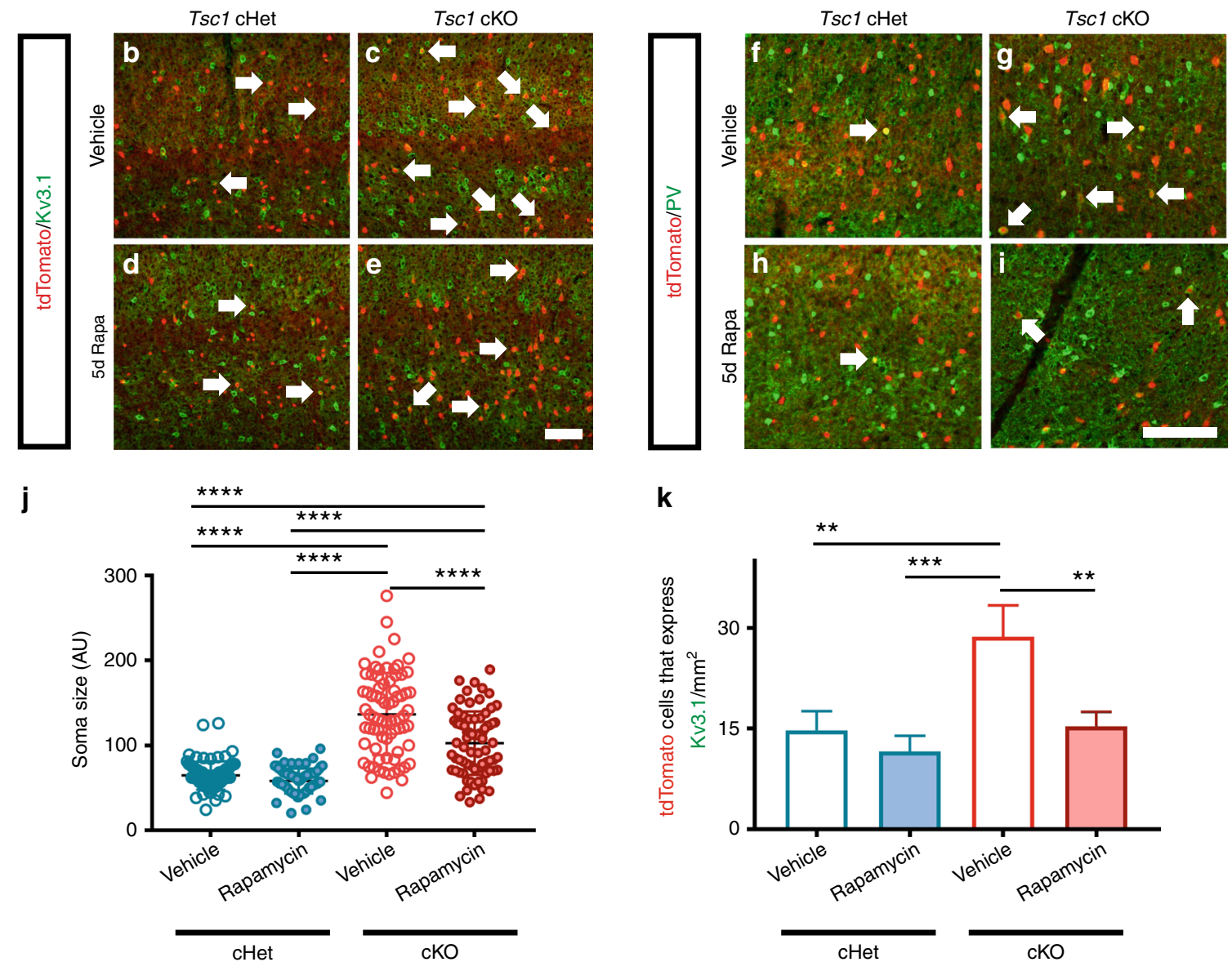

$\mathbf{k}$
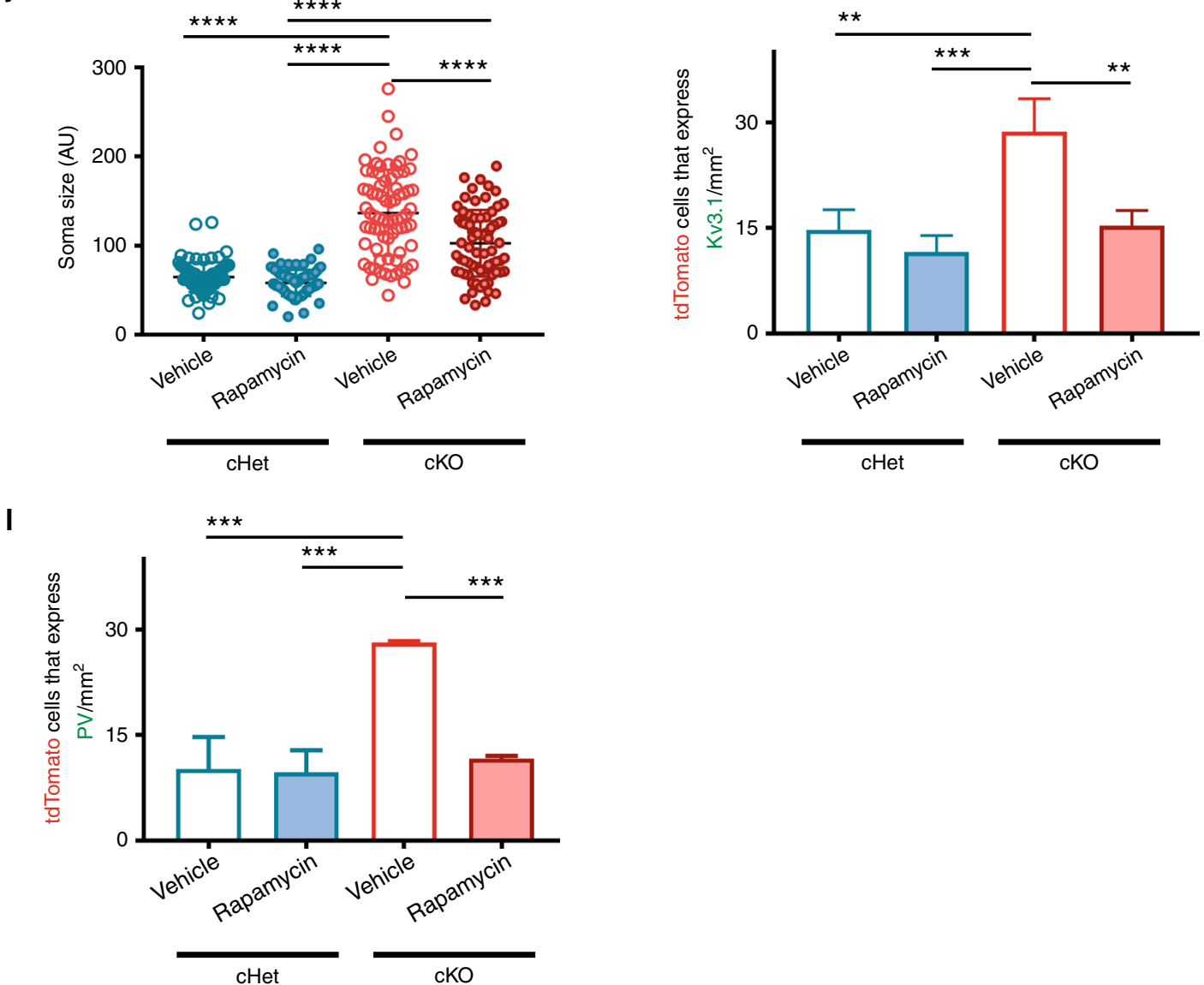

expressed in SST + CINs but not in PV + CINs, as well as TFs that can alter the ratios of SST + and $\mathrm{PV}+\mathrm{CINs}^{22-24,42,53-55}$. Of note, the majority of uniquely expressed TFs are found in SST+ $\mathrm{CINs}^{56}$. One hypothesis is that PV+CIN identity is a default state of MGE-derived CINs, and is repressed by TFs restricted to SST-lineages $^{56}$. Furthermore, perhaps during the maturation of

PV-lineage CINs, MTOR activity is induced to promote PV expression and FS properties, i.e. when PV expression first becomes evident during adolescence. This may be achieved as a consequence of activating certain growth factor receptors or potentially via synaptic activity 7,57 in prospective PV-lineages. However, given that Tsc1/2 and other components of the MTOR 
Fig. 8 Rapamycin decreases PV and Kv3.1 expression in SST-Cre-lineage CINs of cKO mice. a Eight-week old mice that were either SST-Cre; TsC1 cHets or cKOs were injected with vehicle or rapamycin consecutively for 5 days and then assessed a day after for soma size, Kv3.1 and PV expression.

b Quantification of tdTomato+ cell soma size: One-way ANOVA $\left(F_{3,296}=95.39, P<0.0001 ; \mathrm{cKO}\right.$ vs. all groups, $\mathrm{cKO}$ veh vs. rapa, and cHet rapa vs. cKO rapa, $p<0.0001$ ), $n=3$ mice per group (25 cells measured from each). (AU) arbitrary units. Immunofluorescent images of the neocortex from vehicle treated $(\mathbf{c}, \mathbf{d})$ and rapamycin treated $(\mathbf{e}, \mathbf{f})$ cHet and cKO mice that were co-labeled for tdTomato and Kv3.1. Immunofluorescent images of vehicle treated $(\mathbf{g}, \mathbf{h})$ and rapamycin treated $(\mathbf{i}, \mathbf{j}) \mathrm{cHet}$ and cKO mice co-labeled for tdtomato and PV. Arrows point to co-labeled cells. $\mathbf{k}$ Quantification of the cell density of tdTomato+ cells that co-express Kv3.1: One-way ANOVA $\left(F_{3,8}=17.56, P=0.0007\right.$; $c K O$ veh vs. cHet veh, $p=0.0027, \mathrm{cKO}$ veh vs. cHet rapa, $p=$ 0.0007 , and cKO veh vs. cKO rapa, $p=0.0035), n=3$ mice per group. I Quantification of the cell density of tdTomato+cells that co-express PV: One-way ANOVA $\left(F_{3,8}=30.51, P=0.0001 ; c K O\right.$ veh vs. cHet veh and cHet rapa, $p=0.0002$ and $c K O$ veh vs. $\left.c K O, p=0.0004\right), n=3$ mice per group. Data are presented as mean \pm SD in (c) and \pm SEM in all other graphs. ${ }^{\star} p<0.05,{ }^{\star \star} p<0.01,{ }^{\star \star \star \star} p<0.0001$. Scale bar $\mathbf{j}=100 \mu \mathrm{m}$. Source data are provided as a Source Data file

a

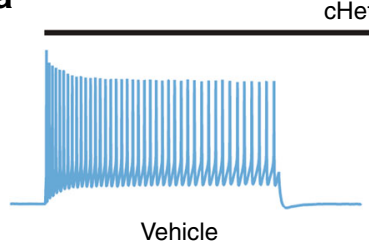

$\mathrm{cHet}$

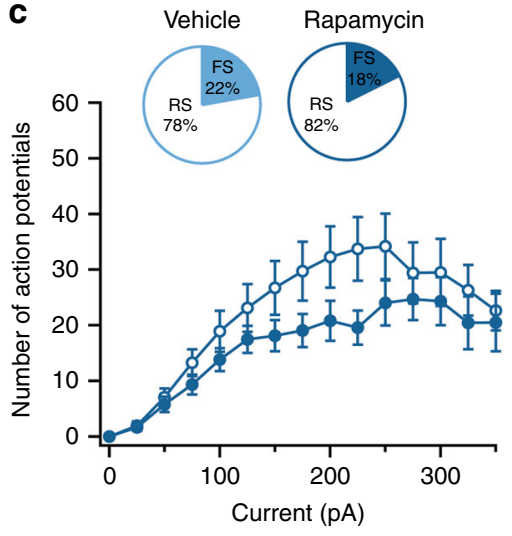

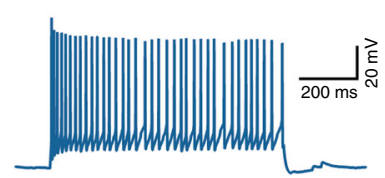

Rapamycin b

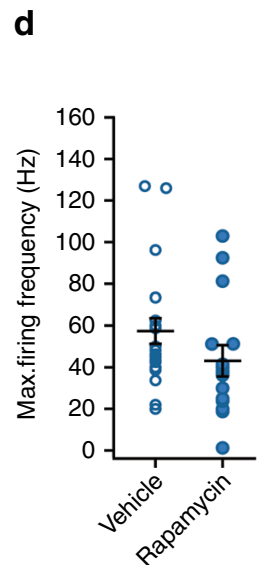

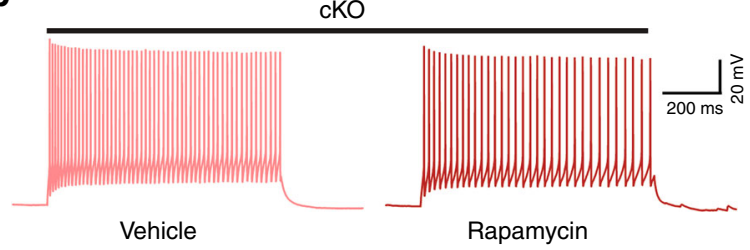

e

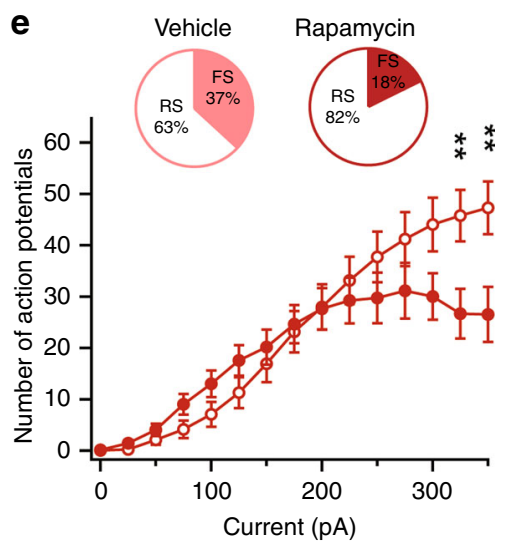

f

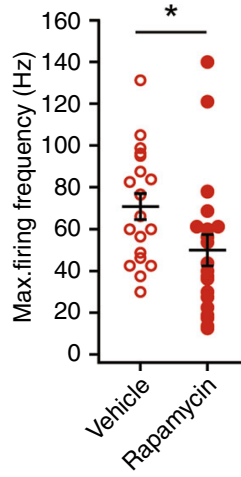

Fig. 9 Rapamycin treatment decreases fast-spiking phenotype in SST-Cre lineage CINs in cKO mice. a, b Example voltage traces in response to suprathreshold current injections in SST+ CINs from vehicle and rapamycin treated cHet (a) and cKO (b) mice. c, d Comparison of firing output: Two-way ANOVA $\left(F_{14,475}=0.63, P=0.83\right)$; and maximum firing frequency: Two-way unpaired $t$-test $\left(t_{33}=1.4, p=0.15\right)$ of tdTomato + CINs in vehicle and rapamycin treated cHet mice (Vehicle, $n=18$ from 2 mice; Rapamycin, $n=17$ cells from 2 mice). Inset, pie charts showing the proportion of SST+ CINs with RS and FS physiological properties in each treatment group. e, $\mathbf{f}$ Same as $(\mathbf{c}, \mathbf{d})$ for vehicle and rapamycin treated cKO mice. Note the CINs from rapamycin treated $\mathrm{cKO}$ mice have lower firing output: Two-way ANOVA with Bonferroni's test $\left(F_{14,555}=2.86, P=0.003 ; 325 \mathrm{pA}, p=0.004\right.$ and $350 \mathrm{pA}$, $p=0.001)$, and maximum firing frequency: Two-way unpaired $t$-test $\left(t_{37}=2.1, p=0.04\right)$; (Vehicle, $n=19$ cells from 2 mice; Rapamycin, $n=20$ from 2 mice). Inset, pie charts showing the proportion of SST-lineage CINs with FS and RS physiological properties in each treatment group. Data are presented as mean \pm SEM in all graphs. ${ }^{\star} p<0.05,{ }^{\star \star} p<0.01,{ }^{\star \star \star \star} p<0.0001$. Source data are provided as a Source Data file

pathway are expressed in most cell types, there would need to be unique factors coupling MTOR signaling in PV-lineages that are different in SST-lineages. These ideas will be investigated in future studies.

Most PV + CINs have FS physiological properties. In contrast, most SST + CINs exhibit a RS physiology, although a class of quasi-fast-spiking SST + neurons was recently described ${ }^{48}$. Moreover, these two neuronal groups are believed to play distinct and dissociable roles in circuit function ${ }^{58-62}$. Our data suggest new possibilities about these classes. First, CINs exhibit intermediate properties after $T s c 1$ loss, which fall along a continuum between the classic SST + and PV+ distinct groups of CINs. Second, the programming of these classes may be more dynamic than previously thought. In particular, rapamycin was able to reverse the expression of PV and FS properties in mature SSTlineage cKO CINs, suggesting that some aspects of CIN cell characteristics and programming are malleable by signalingdependent plasticity mechanisms that remain to be elucidated.

The extent to which changes in molecular (expression of PV vs. SST) and physiological (FS vs.RS) properties correlate with changes in cortical function remains a major question. Differential expression of ion-channels, like Kv3 channels, underlie the fast-spiking properties of PV+CINs ${ }^{20,63}$. Loss of Tsc1 leads to an increase in the occurrence of dual identity CINs, which express both PV and SST. Furthermore, SST-lineage CINs lacking Tsc1 have increased expression of Kv3.1 and the properties of these CINs are shifted towards a FS physiology. Interestingly, deletion of FMRP, another monogenic ASD-risk gene, inhibits protein 
translation downstream of MTOR, of which the Kv3.1 channel is known to be a target in auditory brain stem neurons ${ }^{64}$. These converging findings suggest that modulators of the Kv3.1 channel could be potential targets for treating cellular and circuit abnormalities in TSC. Future work will be necessary to understand whether Tsc1 deletion induced shifts in CIN cell programming underlie behavioral abnormalities in mutant mice and humans diagnosed with TSC, which may lead to new therapeutic targets.

We previously found some overlapping phenotypes after deletion of Pten in MGE progenitors using Nkx2.1-Cre ${ }^{25}$. However, due to a drastic loss in CIN numbers, we were not able to determine if manipulation of MTOR activity, via Pten loss, specifically altered CIN programming. Furthermore, Pten deletion using SST-Cre resulted in no phenotypes, whereas Tsc1 deletion using SST-Cre did exhibit molecular and physiological phenotypes without any loss of CINs. These phenotypes allowed us to gain insights into how MTOR activity influences CIN programming. We do not currently understand why Tscl cKOs in immature SST CINs, but not Pten cKOs, resulted in these phenotypes. However, the Tsc genes lie downstream of Pten and at a crucial point in the signaling pathway inhibiting MTOR signaling. Moreover, they can be inhibited by other signaling events, i.e. MAPK signaling. Thus, both their proximity to MTOR in the signaling cascade and their ability to be inhibited by other signaling moieties may have resulted in differences between the Tsc1 and Pten cKOs.

In summary, we provided evidence that $T s c 1$-inhibition of MTOR represses PV+/FS properties in a cohort of SST-Crelineage CINs. Notably, it suggests that regulation of some CIN molecular and physiological properties can be initiated by nontranscriptional events, i.e. MTOR signaling. However, whether transcription is targeted downstream of MTOR signaling is not yet known. Moreover, we identified specific alterations in GABAergic CINs and underlying molecular mediators (e.g., MTOR and Kv3.1) that could plausibly contribute to neurocognitive symptoms of TSC. Future studies should assess whether similar abnormalities occur in humans diagnosed with TSC and/ or can be reversed by rapamycin analogs, to more directly implicate these findings about CINs in the pathogenesis and/or treatment of TSC.

\section{Methods}

Animals. All mouse strains have been published: Ai14 Cre-reporter ${ }^{29}$, Nkx2.1-

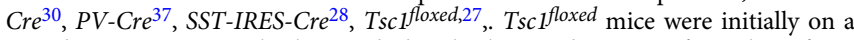
mixed C57BL6/J, CD-1 background, then backcrossed to CD-1 for at least four generations before analysis. For timed pregnancies, noon on the day of the vaginal plug was counted as embryonic day 0.5 . Both male and female mice were assessed. We did not observe any phenotypic differences between genders and combined all data for each genotype. All animal care and procedures were performed and approved according to the Michigan State University and University of California San Francisco Laboratory Animal Research Center guidelines. We have complied with all relevant ethical regulations for animal research.

Cell Counting. To determine cell density $\left(\right.$ cells $/ \mathrm{mm}^{2}$ ), we counted the number of cells in a given section and then divided by the area of that region. To calculate the $\%$ of tdTomato ${ }^{+}$cells that co-labeled with specific markers, we divided the number of co-labeled cells by the total number of tdTomato ${ }^{+}$cells. For cell transplants, all tdTomato $^{+}$cells were counted in the neocortex from all sections in a rostral to caudal series. For cell fate counts, only transplants where at least 50 tdTomato ${ }^{+}$ cells could be counted were used for analysis. For soma size quantification, the perimeter of each tdTomato ${ }^{+}$cell's soma was traced in Image J, and at least 25 cells were measured and averaged for each mouse.

Acute cortical slice preparation. Adult mice of either sex (P45-P60 days) were anesthetized with an intraperitonial injection of euthasol and transcardially perfused with an ice-cold cutting solution containing (in mM) 210 sucrose, $2.5 \mathrm{KCl}$, $1.25 \mathrm{NaH}_{2} \mathrm{PO}_{4}, 25 \mathrm{NaHCO}_{3}, 0.5 \mathrm{CaCl}_{2}, 7 \mathrm{MgCl}_{2}, 7$ dextrose (bubbled with 95\% $\mathrm{O}_{2}-5 \% \mathrm{CO}_{2}, \mathrm{pH} \sim 7.4$ ). Mice were decapitated, the brains were removed and two parallel cuts were made along the coronal plane at the rostral and caudal ends of the brains. Brains were mounted on the flat surface created at the caudal end. Approximately, three coronal slices $(250 \mu \mathrm{m}$ thick) were obtained using Vibrating blade microtome (VT1200S, Leica Microsystems Inc.). Slices were allowed to recover at $34^{\circ} \mathrm{C}$ for $30 \mathrm{~min}$ followed by $30 \mathrm{~min}$ recovery at room temperature in a holding solution containing (in mM) $125 \mathrm{NaCl}, 2.5 \mathrm{KCl}, 1.25 \mathrm{NaH}_{2} \mathrm{PO}_{4}, 25$ $\mathrm{NaHCO}_{3}, 2 \mathrm{CaCl}_{2}, 2 \mathrm{MgCl}_{2}, 12.5$ dextrose, 1.3 ascorbic acid, 3 sodium pyruvate.

Whole-cell patch clamp recordings. Somatic whole-cell current clamp and voltage clamp recordings were obtained from submerged slices perfused in heated $\left(32-34^{\circ} \mathrm{C}\right)$ artificial cerebrospinal fluid (aCSF) containing (in $\left.\mathrm{mM}\right) ; 125 \mathrm{NaCl}, 3$ $\mathrm{KCl}, 1.25 \mathrm{NaH}_{2} \mathrm{PO}_{4}, 25 \mathrm{NaHCO}_{3}, 2 \mathrm{CaCl}_{2}, 1 \mathrm{MgCl}_{2}, 12.5$ dextrose (bubbled with $\left.95 \% \mathrm{O}_{2} / 5 \% \mathrm{CO}_{2}, \mathrm{pH} \sim 7.4\right)$. Neurons were visualized using DIC optics fitted with a 40x water-immersion objective (BX51WI, Olympus microscope). Pyramidal neurons and tdtomato expressing CINs located in layer 5 were targeted for patching. Patch electrodes (2-4 M $\Omega$ ) were pulled from borosilicate capillary glass of external diameter $1 \mathrm{~mm}$ (Sutter Instruments) using a Flaming/Brown micropipette puller (model P-2000, Sutter Instruments). For current clamp recordings, electrodes were filled with an internal solution containing the following (in $\mathrm{mM}$ ): $120 \mathrm{~K}$-gluconate $20 \mathrm{KCl}, 10 \mathrm{HEPES}, 4 \mathrm{NaCl}, 7 \mathrm{~K}_{2}$-phosphocreatine, $0.3 \mathrm{Na}-\mathrm{GTP}$, and $4 \mathrm{Mg}$-ATP ( $\mathrm{pH} \sim 7.3$ adjusted with $\mathrm{KOH}$ ). Biocytin (Vector Laboratories) was included (0.1-0.2\%) for subsequent histological processing. For voltage clamp recordings, the internal solution contained the following (in $\mathrm{mM}$ ): $130 \mathrm{Cs}$-methanesulfonate, $10 \mathrm{CsCl}, 10 \mathrm{HEPES}, 4 \mathrm{NaCl}, 7$ phosphocreatine, $0.3 \mathrm{Na}-\mathrm{GTP}, 4 \mathrm{Mg}$-ATP, and 2 QX314-Br ( $\mathrm{pH} \sim 7.3$ adjusted with $\mathrm{CsOH}$ ).

Electrophysiology data were recorded using Multiclamp 700B amplifier (Molecular Devices). Voltages have not been corrected for measured liquid junction potential $(\sim 8 \mathrm{mV})$. Upon successful transition to the whole-cell configuration, the neuron was given at least $5 \mathrm{~min}$ to stabilize before data were collected. Series resistance and pipette capacitance were appropriately compensated before each recording. Series resistance was usually 10-20 M $\Omega$, and experiments were terminated if series resistances exceeded $25 \mathrm{M} \Omega$.

Electrophysiology protocols and data analysis. All data analyses were performed using custom routines written in IGOR Pro (Wavemetrics). Code is available upon request. Resting membrane potential (RMP) was measured as the membrane voltage measured in current clamp mode immediately after reaching the whole-cell configuration. Input resistance (Rin) was calculated as the slope of the linear fit of the voltage-current plot generated from a family of hyperpolarizing and depolarizing current injections ( -50 to $+20 \mathrm{pA}$, steps of $10 \mathrm{pA})$. Firing output was calculated as the number of action potentials (APs) fired in response to $800 \mathrm{~ms}$ long depolarizing current injections $(25-500 \mathrm{pA})$. Firing frequency was calculating as the number of APs fired per second. Rheobase was measured as the minimum current injection that elicited spiking. Firing traces in response to $50 \mathrm{pA}$ current above the rheobase were used for analysis of single AP properties- AP threshold, maximum $d V / d t$ (rate of rise of AP), AP amplitude, AP half-width and fast after hyperpolarization (fAHP) amplitude. Threshold was defined as the voltage at which the value of third derivative of voltage with time is maximum. Action potential amplitude was measured from threshold to peak, with the half-width measured at half this distance. Fast after hyperpolarization was measured from the threshold to the negative voltage peak after the AP. Index of spike-frequency accommodation (SFA) was calculated as the ratio of the last inter-spike interval to the first inter-spike interval. Coefficient of variance $(\mathrm{CV})$ for inter-spike interval (ISI), AP amplitude and AP half-width was calculated as the ratio of standard deviation to the mean. Recorded CINs in all genotypes were classified as fastspiking or regular-spiking based on electrophysiological properties. Specifically, CINs were classified as fast-spiking if the AP half-width was $<0.5 \mathrm{~ms}$, firing frequency $>50 \mathrm{~Hz}$, fAHP amplitude was $>14 \mathrm{mV}$ and SFA was $<2$. The correspondence between PV expression and fast-spiking electrophysiological properties was confirmed in a subset of CINs by quantification of PV staining in biocytin-filled neurons (16 of 21 biocytin-labeled CINs classified as fast-spiking were PV+).

Spontaneous inhibitory currents were recorded for $5 \mathrm{~min}$ with neurons voltage clamped at $+10 \mathrm{mV}$. Miniature inhibitory currents were recorded in the presence of TTX. Spontaneous and miniature inhibitory currents were analyzed off-line using Clampfit (pClamp, Molecular devices) event detection.

For Cre-dependent expression, $600 \mathrm{nl}$ of $A A V$-DIO-ChR2-eFYP virus was bilaterally injected into P30-40 SST-Cre mice. We waited at least 4 weeks after virus injection before preparing brain slices. To measure optogenetically evoked inhibitory currents (oIPSCs), we voltage-clamped layer 5 pyramidal neurons at $+10 \mathrm{mV}$. We stimulated ChR2 using $5 \mathrm{~ms}$ long single or multiple light pulses (maximum light power, $4 \mathrm{~mW} / \mathrm{mm}^{2}$ ) generated by a Lambda DG-4 high-speed optical switch with a $300 \mathrm{~W}$ Xenon lamp (Sutter Instruments) and an excitation filter centered around $470 \mathrm{~nm}$, delivered to the slice through a $\times 40$ objective (Olympus). Frequency dependent attenuation $(20 \mathrm{~Hz})$ and paired-pulse ratios of oIPSCs were measured at the maximum light power.

To measure unitary inhibitory currents (uIPSCs), dual whole-cell recordings were obtained from connected layer 5 tdTomato expressing (SST-Cre lineage or $P V$-Cre lineage) CINs and adjacent layer 5 pyramidal neurons. We obtained current clamp recordings from tdTomato + CINs and voltage clamp recordings $(+10 \mathrm{mV})$ from pyramidal neurons. Short duration $(1-2 \mathrm{~ms})$ depolarizing current steps $(1-1.5 \mathrm{nA})$ were injected into the CINs to elicit single action potentials and 
uIPSCs were recorded in postsynaptic pyramidal neurons. Average of 20-30 uIPSCs were used to calculate the peak amplitude (from the baseline to the peak of uIPSC), rising slope (slope of a fitting line from 10-90\% after onset of uIPSC) and total charge of uIPSCs (integral of uIPSCs from the onset until the trace returned completely to the baseline).

Computer simulations. Simulations were performed using the NEURON 7.4 simulation environment (Carnevale and Hines, 2006) with Python interface. Four different morphologically detailed models of SST + neocortical layer 5 CINs were used from Allen Brain Atlas; Allen Institute (2015) Documentation, http:// help.brain-map.org/display/celltypes/Documentation. Model files for simulation in Python environment were downloaded from ModelDB (senselab.med.yale.edu). Maximum conductance densities (in S/ $\left.\mathrm{cm}^{2}\right)$ of Kv3.1 channel $(\overline{\mathrm{g}} \mathrm{kv} 3.1)$ were noted for the four different models $(0.25,0.009,0.29,0.56)$. These values were used for the low Kv3.1 conductance conditions. To simulate the effects of increase in Kv3.1 expression, $\overline{\mathrm{g}}$ kv3.1 was increased to 1.5 in all four models. Firing output in the low and high Kv3.1 conditions were measured as the number of spikes fired in response to $1 \mathrm{~s}$ long depolarizing current steps (50-350 pA). Spike times were measured by determining the time of AP peak. Instantaneous frequency (1/inter-spike interval) was computed from current injections that elicited $>40$ APs. Subthreshold membrane properties were calculated from the voltage traces in response to hyperpolarizing and depolarizing current injections $(-50$ to $+20 \mathrm{pA}$, steps of $10 \mathrm{pA})$.

Immunofluorescent tissue staining. Immunofluorescent labeling was performed on $25 \mu \mathrm{m}$ cryo-sectioned tissue with the following antibodies: mouse anti-Kv3.1b 1:400 (NeuroMab cat. \# 75-041); rabbit anti-phosphoS6 ${ }^{\text {SER240/244 }}$ 1:400 (Cell Signaling Technologies cat. \# 5364); rabbit anti-PV 1:400 (Swant cat. \# PV 27); rat anti-SST 1:200 (Millipore cat. \# MAB354). The appropriate 488, 594 or 647 Alexaconjugated secondary antibodies were from Thermo Fisher Scientific (1:300 dilution). Sections were cover-slipped with Vectashield (Vector labs).

MGE cell transplantation. Lentiviral transduction of MGE cells followed by intracranial transplantation followed a reported protocol ${ }^{40}$. Briefly, HEK293T cells were transfected using Lipofectamine2000 (Thermo Fischer Scientific) with four plasmids to generate lentivirus particles. E13.5 MGEs from individual embryos were dissected in ice-cold HBSS and then kept on ice in DMEM media (containing $10 \%$ fetal bovine serum). MGEs were then mechanically dissociated with a p1000 pipette tip and transduced with lentivirus. To perform transductions, dissociated MGE cells were mixed with pre-warmed media that was at physiological $\mathrm{pH}$ and was incubated with $\sim 10-20 \mu \mathrm{ls}$ of concentrated lentivirus, with titers ranging from $1 \times 10^{8}-1 \times 10^{10}$ infectious units $/ \mathrm{ml}$. They were next incubated at $37^{\circ} \mathrm{C}$ for $30 \mathrm{~min}$, with intervals of agitation. Since the MGE cells had a Cre-dependent reporter, $A i 14^{29}$, only MGE cells transduced with Cre-expressing lentiviruses were visible in the host neocortex after transplantation. This combinatorial method allows the transduced/transplanted cells to express a strong reporter, i.e. tdTomato expressed from the beta-actin promoter, while maintaining cell type specificity. Cells were then pelleted in a tabletop centrifuge at low speed $(700 \times g, \sim 4 \mathrm{~min})$ and washed 3 times with media followed by trituration to disperse cells between each wash to remove excess virus. The final pellet was left under a few $\mu$ ls of media, put on ice, and the remaining media was removed with a fine point kim wipe before the injection needle was loaded. For injections, a glass micropipette with a $45^{\circ}$ beveled tip, $\sim 50 \mu \mathrm{m}$ outer diameter, was preloaded with sterile mineral oil and cells were then front-loaded into the tip of the needle using a plunger connected to a hydraulic drive (Narishige MO-10) that was mounted to a stereotaxic frame. Pups were anesthetized on ice for 1-2 min before being placed on a mold for injections. Each pup received 3-6 injections of cells, at $70 \mathrm{nl}$ per site, in their right hemisphere. These sites were about $1 \mathrm{~mm}$ apart from rostral to caudal and medial to lateral and were then injected into layers V-VI of a P1 WT neocortex. After injections, pups were allowed to recover and then put back with their mother. The transplanted mice were sacrificed at 35 days post-transplant and transcardially perfused with PBS followed by $4 \%$ PFA. Brains were then post-fixed in 4\% PFA for a short interval, $30 \mathrm{~min}$, and then sunk in 30\% sucrose before embedding in OCT.

DNA vector generation. The $D l x I 12 b-B G-I R E S$-Cre lentiviral vector backbone is reported in 65 . To generate the DlxI12b-BG-hTSC1-IRES-Cre lentiviral vector, the human TSC1 gene was PCR amplified from a vector containing the $h T S C 1$ cDNA (Addgene) ${ }^{66}$. The primers (Forward 5'-GAGAGCTAGCATGGCCCAACAAGCA AATG-3' and Reverse 5'-GAGATGTACATTAGCTGTGTTCATGATG-3'), were used to introduce NheI and BsrGI restriction enzyme sites, respectively (underlined). Next the PCR product and the DlxI12b-BG-IRES-Cre vector were digested with the appropriate enzymes and ligated. The vector was verified by restriction digest and sequencing.

Rapamycin treatment. Rapamycin (LC Laboratories, Woburn, MA, USA) was dissolved in ethanol at a concentration of $20 \mathrm{mg} / \mathrm{ml}$ and stored at $-20^{\circ} \mathrm{C}$. On the day of the injections, drug was resuspended in saline (containing $0.25 \%$ PEG and $0.25 \%$ Tween-80). Mice received rapamycin solution $\left(10 \mathrm{mg} \mathrm{kg}^{-1}\right)$ or an equal volume of vehicle by intraperitoneal injection once daily for 5 consecutive days as previously described ${ }^{67}$
Quantification and statistical analysis. For anatomical analyses, statistics were performed using Prism version 6 , a $p$-value of $<0.05$ was considered significant. For most parametric measurements, we used a One-Way ANOVA with Tukey's post test to determine significance (cell density and soma size measurements). For nonparametric data sets, we used a Chi-squared test with Yate's correction to determine significance (data normalized and expressed as a percentage). For electrophysiological analyses, statistical comparisons were done using custom-written software in MATLAB or Prism (Graphpad). Statistical significance was assessed using a paired or unpaired two-tailed Student's $t$-test. For repeated observations, two-way ANOVA followed by a post hoc Tukey's or Bonferroni's test was used. Statistical comparisons across three groups were done using one-way ANOVA followed by a post hoc Tukey test. Probability values for ANOVAs are denoted as $P$ and the probability values for post hoc comparisons are denoted as $p$. Error bars indicate \pm SEM unless otherwise noted.

Reporting summary. Further information on research design is available in the Nature Research Reporting Summary linked to this article.

\section{Data availability}

The data used in the analysis of this study are available from the corresponding authors upon reasonable request. A reporting summary for this article is available as a Supplementary Information file. The source data for all relevant figures are provided as a Source Data file.

Received: 13 April 2019; Accepted: 4 October 2019; Published online: 01 November 2019

\section{References}

1. Chu-Shore, C. J., Major, P., Camposano, S., Muzykewicz, D. \& Thiele, E. A The natural history of epilepsy in tuberous sclerosis complex. Epilepsia 51, 1236-1241 (2010).

2. Numis, A. L. et al. Identification of risk factors for autism spectrum disorders in tuberous sclerosis complex. Neurology 76, 981-987 (2011).

3. Spurling Jeste, S. et al. Early developmental trajectories associated with ASD in infants with tuberous sclerosis complex. Neurology 83, 160-168 (2014).

4. Winterkorn, E. B., Pulsifer, M. B. \& Thiele, E. A. Cognitive prognosis of patients with tuberous sclerosis complex. Neurology 68, 62-64 (2007).

5. European Chromosome 16 Tuberous Sclerosis Consortium. Identification and characterization of the tuberous sclerosis gene on chromosome 16. Cell 75, 1305-1315 (1993)

6. van Slegtenhorst, M. et al. Interaction between hamartin and tuberin, the TSC1 and TSC2 gene products. Hum. Mol. Genet 7, 1053-1057 (1998).

7. Laplante, M. \& Sabatini, D. M. mTOR signaling in growth control and disease. Cell 149, 274-293 (2012).

8. O'Roak, B. J. et al. Multiplex targeted sequencing identifies recurrently mutated genes in autism spectrum disorders. Science 338, 1619-1622 (2012).

9. Richards, C., Jones, C., Groves, L., Moss, J. \& Oliver, C. Prevalence of autism spectrum disorder phenomenology in genetic disorders: a systematic review and meta-analysis. Lancet Psychiatry 2, 909-916 (2015).

10. Curatolo, P., Moavero, R. \& de Vries, P. J. Neurological and neuropsychiatric aspects of tuberous sclerosis complex. Lancet Neurol. 14, 733-745 (2015).

11. Sohal, V. S. \& Rubenstein, J. L. R. Excitation-inhibition balance as a framework for investigating mechanisms in neuropsychiatric disorders. Mol. Psychiatry. https://doi.org/10.1038/s41380-019-0426-0. (2019).

12. Bateup, H. S., Takasaki, K. T., Saulnier, J. L., Denefrio, C. L. \& Sabatini, B. L. Loss of Tsc1 in vivo impairs hippocampal mGluR-LTD and increases excitatory synaptic function. J. Neurosci. 31, 8862-8869 (2011).

13. Ehninger, D. et al. Reversal of learning deficits in a Tsc2+/- mouse model of tuberous sclerosis. Nat. Med 14, 843-848 (2008).

14. Tavazoie, S. F., Alvarez, V. A., Ridenour, D. A., Kwiatkowski, D. J. \& Sabatini, B. L. Regulation of neuronal morphology and function by the tumor suppressors Tsc1 and Tsc2. Nat. Neurosci. 8, 1727-1734 (2005).

15. Wonders, C. P. \& Anderson, S. A. The origin and specification of cortical interneurons. Nat. Rev. Neurosci. 7, 687-696 (2006).

16. Miyoshi, G. et al. Genetic fate mapping reveals that the caudal ganglionic eminence produces a large and diverse population of superficial cortical interneurons. J. Neurosci. 30, 1582-1594 (2010).

17. Kepecs, A. \& Fishell, G. Interneuron cell types are fit to function. Nature 505, 318-326 (2014).

18. Huang, Z. J., Di Cristo, G. \& Ango, F. Development of GABA innervation in the cerebral and cerebellar cortices. Nat. Rev. Neurosci. 8, 673-686 (2007).

19. Chiu, C. Q. et al. Compartmentalization of GABAergic inhibition by dendritic spines. Science 340, 759-762 (2013). 
20. Erisir, A., Lau, D., Rudy, B. \& Leonard, C. S. Function of specific K(+) channels in sustained high-frequency firing of fast-spiking neocortical interneurons. J. Neurophysiol. 82, 2476-2489 (1999).

21. Butt, S. J. B. et al. The requirement of Nkx2-1 in the temporal specification of cortical interneuron subtypes. Neuron 59, 722-732 (2008).

22. Liodis, P. et al. Lhx6 activity is required for the normal migration and specification of cortical interneuron subtypes. J. Neurosci. 27, 3078-3089 (2007).

23. Zhao, Y. et al. Distinct molecular pathways for development of telencephalic interneuron subtypes revealed through analysis of Lhx6 mutants. J. Comp. Neurol. 510, 79-99 (2008).

24. Pai, E. L.-L. et al. Mafb and c-Maf Have Prenatal Compensatory and Postnatal Antagonistic Roles in Cortical Interneuron Fate and Function. Cell Rep. 26, 1157-1173.e5 (2019).

25. Vogt, D., Cho, K. K. A., Lee, A. T., Sohal, V. S. \& Rubenstein, J. L. R. The parvalbumin/somatostatin ratio is increased in Pten mutant mice and by human PTEN ASD alleles. Cell Rep. 11, 944-956 (2015).

26. Wong, F. K. et al. Pyramidal cell regulation of interneuron survival sculpts cortical networks. Nature 557, 668-673 (2018).

27. Kwiatkowski, D. J. et al. A mouse model of TSC1 reveals sex-dependent lethality from liver hemangiomas, and up-regulation of p70S6 kinase activity in Tsc1 null cells. Hum. Mol. Genet 11, 525-534 (2002).

28. Taniguchi, H. et al. A Resource of Cre driver lines for genetic targeting of GABAergic neurons in cerebral cortex. Neuron 71, 995-1013 (2011).

29. Madisen, L. et al. A robust and high-throughput Cre reporting and characterization system for the whole mouse brain. Nat. Neurosci. 13, 133-40. (2010).

30. Xu, Q., Tam, M. \& Anderson, S. A. Fate mapping Nkx2.1-lineage cells in the mouse telencephalon. J. Comp. Neurol. 506, 16-29 (2008).

31. Halabisky, B., Shen, F., Huguenard, J. R. \& Prince, D. A. Electrophysiological classification of somatostatin-positive interneurons in mouse sensorimotor cortex. J. Neurophysiol. 96, 834-845 (2006).

32. Butt, S. J. B. et al. The temporal and spatial origins of cortical interneurons predict their physiological subtype. Neuron 48, 591-604 (2005).

33. Ma, Y., Hu, H., Berrebi, A. S., Mathers, P. H. \& Agmon, A. Distinct subtypes of somatostatin-containing neocortical interneurons revealed in transgenic mice. J. Neurosci. 26, 5069-5082 (2006).

34. Du, J., Zhang, L., Weiser, M., Rudy, B. \& McBain, C. J. Developmental expression and functional characterization of the potassium-channel subunit $\mathrm{Kv} 3.1 \mathrm{~b}$ in parvalbumin-containing interneurons of the rat hippocampus. $J$. Neurosci. 16, 506-518 (1996).

35. Gouwens, N. W. et al. Systematic generation of biophysically detailed models for diverse cortical neuron types. Nat. Commun. 9, 710 (2018).

36. Safari, M.-S., Mirnajafi-Zadeh, J., Hioki, H. \& Tsumoto, T. Parvalbuminexpressing interneurons can act solo while somatostatin-expressing interneurons act in chorus in most cases on cortical pyramidal cells. Sci. Rep. 7, 12764 (2017).

37. Hippenmeyer, S. et al. A developmental switch in the response of DRG neurons to ETS transcription factor signaling. PLoS Biol. 3, e159 (2005).

38. Cohen, S. M. et al. Excitation-transcription coupling in parvalbumin-positive interneurons employs a novel CaM kinase-dependent pathway distinct from excitatory neurons. Neuron 90, 292-307 (2016).

39. Parrish, R. R., Codadu, N. K., Racca, C. \& Trevelyan, A. J. Pyramidal cell activity levels affect the polarity of activity-induced gene transcription changes in interneurons. J. Neurophysiol. 120, 2358-2367 (2018).

40. Vogt, D. et al. Viral-mediated labeling and transplantation of medial ganglionic eminence (MGE) cells for in vivo studies. J. Vis. Exp. 98, e52740 (2015).

41. Arguello, A. et al. Dapper antagonist of catenin-1 cooperates with Dishevelled1 during postsynaptic development in mouse forebrain GABAergic interneurons. PloS One 8, e67679 (2013).

42. Vogt, D. et al. Lhx6 directly regulates Arx and CXCR7 to determine cortical interneuron fate and laminar position. Neuron 82, 350-364 (2014).

43. Ajram, L. A. et al. Shifting brain inhibitory balance and connectivity of the prefrontal cortex of adults with autism spectrum disorder. Transl. Psychiatry 7, e1137 (2017).

44. Han, S. et al. Autistic-like behaviour in Scnla $+/-$ mice and rescue by enhanced GABA-mediated neurotransmission. Nature 489, 385-390 (2012).

45. Markram, K., Rinaldi, T., La Mendola, D., Sandi, C. \& Markram, H. Abnormal fear conditioning and amygdala processing in an animal model of autism. Neuropsychopharmacol 33, 901-912 (2008).

46. Antoine, M. W., Langberg, T., Schnepel, P. \& Feldman, D. E. Increased excitation-inhibition ratio stabilizes synapse and circuit excitability in four autism mouse models. Neuron 101, 648-661.e4 (2019).

47. $\mathrm{Fu}, \mathrm{C}$. et al. GABAergic interneuron development and function is modulated by the Tsc1 gene. Cereb. Cortex 22, 2111-2119 (2012).
48. Nigro, M. J., Hashikawa-Yamasaki, Y. \& Rudy, B. Diversity and connectivity of layer 5 somatostatin-expressing interneurons in the mouse barrel cortex. J. Neurosci. 38, 1622-1633 (2018).

49. Paul, A. et al. Transcriptional architecture of synaptic communication delineates GABAergic neuron identity. Cell 171, 522-539.e20 (2017).

50. Tasic, B. et al. Adult mouse cortical cell taxonomy revealed by single cell transcriptomics. Nat. Neurosci. 19, 335-346 (2016).

51. Zhao, J.-P. \& Yoshii, A. Hyperexcitability of the local cortical circuit in mouse models of tuberous sclerosis complex. Mol. Brain 12, 6 (2019).

52. D'Gama, A. M. et al. Somatic mutations activating the mTOR pathway in dorsal telencephalic progenitors cause a continuum of cortical dysplasias. Cell Rep. 21, 3754-3766 (2017).

53. Mayer, C. et al. Developmental diversification of cortical inhibitory interneurons. Nature 555, 457-462 (2018).

54. Stanco, A. et al. NPAS1 represses the generation of specific subtypes of cortical interneurons. Neuron 84, 940-953 (2014).

55. Hu, J. S. et al. Coup-TF1 and Coup-TF2 control subtype and laminar identity of MGE-derived neocortical interneurons. Dev 144, 2837-2851 (2017).

56. Hu, J. S., Vogt, D., Sandberg, M. \& Rubenstein, J. L. Cortical interneuron development: a tale of time and space. Dev 144, 3867-3878 (2017).

57. Pirbhoy, P. S., Farris, S. \& Steward, O. Synaptically driven phosphorylation of ribosomal protein S6 is differentially regulated at active synapses versus dendrites and cell bodies by MAPK and PI3K/mTOR signaling pathways. Learn Mem. 24, 341-357 (2017).

58. Abbas, A. I. et al. Somatostatin interneurons facilitate hippocampalprefrontal synchrony and prefrontal spatial encoding. Neuron 100, 926-939 (2018).

59. Lee, S.-H. et al. Activation of specific interneurons improves V1 feature selectivity and visual perception. Nature 488, 379-383 (2012).

60. Phillips E. A., Hasenstaub A. R. Asymmetric effects of activating and inactivating cortical interneurons. eLife 2016; 5. https://doi.org/10.7554/ eLife.18383.

61. Phillips, E. A. K., Schreiner, C. E. \& Hasenstaub, A. R. Cortical interneurons differentially regulate the effects of acoustic context. Cell Rep. 20, 771-778 (2017).

62. Pinto, L. \& Dan, Y. Cell-type-specific activity in prefrontal cortex during goaldirected behavior. Neuron 87, 437-450 (2015).

63. Rudy, B. \& McBain, C. J. Kv3 channels: voltage-gated $\mathrm{K}+$ channels designed for high-frequency repetitive firing. Trends Neurosci. 24, 517-526 (2001).

64. Strumbos, J. G., Brown, M. R., Kronengold, J., Polley, D. B. \& Kaczmarek, L. $\mathrm{K}$. Fragile $\mathrm{X}$ mental retardation protein is required for rapid experiencedependent regulation of the potassium channel Kv3.1b. J. Neurosci. 30, 10263-10271 (2010).

65. Vogt, D. et al. Mouse Cntnap2 and human CNTNAP2 ASD alleles cell autonomously regulate $\mathrm{PV}+$ cortical interneurons. Cereb. Cortex 28, 3868-3879 (2018).

66. Tee, A. R. et al. Tuberous sclerosis complex-1 and -2 gene products function together to inhibit mammalian target of rapamycin (mTOR)-mediated downstream signaling. Proc. Natl. Acad. Sci. USA 99, 13571-13576 (2002).

67. Zhou, J. et al. Pharmacological inhibition of mTORC1 suppresses anatomical, cellular, and behavioral abnormalities in neural-specific Pten knock-out mice. J. Neurosci. 29, 1773-1783 (2009).

\section{Acknowledgements}

V.S.S. was funded by CDMRP (\#TS150059) and NIMH R01 (\#MH106507). J.L.R. by Nina Ireland, NIMH R01 (\#MH081880), NIMH R37 (\#MH049428) and CDMRP (\#TS150059). R.M. by NARSAD Young Investigator Award (Leichtung Family Investigator, BBRF). E.L.L.P. by UCSF Neuroscience Program and NIMH R01 (\#MH081880). A.N.R. by NIMH R01 (\#MH081880). A.M.S., K.A., and D.V. by the Spectrum HealthMSU Alliance Corporation.

\section{Author contributions}

R.M. conducted electrophysiology experiments, biophysical simulations, morphological analysis, and virus injections. E.L.L.P. performed FISH, immunofluorescent labeling, and imaging. D.V. performed transplantation assays, immunofluorescence labeling, and imaging. A.M.S. and P.M. performed soma size measurements. A.N.R. performed biocytin immuno-labeling. K.A. performed pS6 staining. R.M., E.L.L.P., J.L.R., V.S.S., and D.V. conceived experiments and wrote the paper, all authors read and edited the PAPER.

\section{Competing interests}

J.L.R. is cofounder, stockholder, and on the scientific board of Neurona, a company studying the potential therapeutic use of CIN transplantation. V.S.S. receives research funding from Neurona. The remaining authors declare no competing interests. 


\section{Additional information}

Supplementary information is avaliable for this paper at https://doi.org/10.1038/s41467019-12962-4

Correspondence and requests for materials should be addressed to V.S.S. or D.V.

Peer review information Nature Communications thanks Stewart Anderson, Timothy Petros and Mustafa Sahin for their contribution to the peer review of this work. Peer reviewer reports are available.

Reprints and permission information is available at http://www.nature.com/reprints

Publisher's note Springer Nature remains neutral with regard to jurisdictional claims in published maps and institutional affiliations. (c) (i) Open Access This article is licensed under a Creative Commons Attribution 4.0 International License, which permits use, sharing, adaptation, distribution and reproduction in any medium or format, as long as you give appropriate credit to the original author(s) and the source, provide a link to the Creative Commons license, and indicate if changes were made. The images or other third party material in this article are included in the article's Creative Commons license, unless indicated otherwise in a credit line to the material. If material is not included in the article's Creative Commons license and your intended use is not permitted by statutory regulation or exceeds the permitted use, you will need to obtain permission directly from the copyright holder. To view a copy of this license, visit http://creativecommons.org/ licenses/by/4.0/.

(C) The Author(s) 2019 